JOURNAL OF THE

AMERICAN MATHEMATICAL SOCIETY

Volume 17, Number 1, Pages 109-153

S 0894-0347(03)00443-0

Article electronically published on September 30, 2003

\title{
ALMOST GLOBAL EXISTENCE FOR QUASILINEAR WAVE EQUATIONS IN THREE SPACE DIMENSIONS
}

\author{
MARKUS KEEL, HART F. SMITH, AND CHRISTOPHER D. SOGGE
}

\section{INTRODUCTION}

This article studies almost global existence for solutions of quadratically quasilinear systems of wave equations in three space dimensions. The approach here uses only the classical invariance of the wave operator under translations, spatial rotations, and scaling. Using these techniques we can handle wave equations in Minkowski space or Dirichlet-wave equations in the exterior of a smooth, starshaped obstacle. We can also apply our methods to systems of quasilinear wave equations having different wave speeds.

This extends our work [11] for the semilinear case. Previous almost global existence theorems for quasilinear equations in three space dimensions were for the non-obstacle case. In [9], John and Klainerman proved almost global existence on Minkowski space for quadratic, quasilinear equations using the Lorentz invariance of the wave operator in addition to the symmetries listed above. Subsequently, in [14, Klainerman and Sideris obtained the same result for a class of quadratic, divergence-form nonlinearities without relying on Lorentz invariance. This line of thought was refined and applied to prove global-in-time results for null-form equations related to the theory of elasticity in Sideris [22], 23], and for multiple-speed systems of null-form quasilinear equations in Sideris and $\mathrm{Tu}$ [24, and Yokoyama 29].

The main difference between our approach and the earlier ones is that we exploit the $O\left(|x|^{-1}\right)$ decay of solutions of wave equations with sufficiently decaying initial data as much as we involve the stronger $O\left(t^{-1}\right)$ decay. Here, of course, $x=\left(x_{1}, x_{2}, x_{3}\right)$ is the spatial component, and $t$ the time component, of a spacetime vector $(t, x) \in \mathbb{R}_{+} \times \mathbb{R}^{3}$. Establishing $O\left(|x|^{-1}\right)$ decay is considerably easier and can be achieved using only the invariance with respect to translations and spatial rotation. A weighted $L^{2}$ space-time estimate for inhomogeneous wave equations (Proposition 3.1] below, from [1]) is important in making the spatial decay useful for the long-time existence argument.

For semilinear systems, one can show almost global existence from small data using only this spatial decay [11]. For quasilinear systems, however, we also have to show that both first and second derivatives of $u$ decay like $1 / t$. Fortunately, we can do this using a variant of some $L^{1} \rightarrow L^{\infty}$ estimates of John, Hörmander,

Received by the editors September 16, 2002.

2000 Mathematics Subject Classification. Primary 35L05, 35L10, 35L15, 35L20, 35L70.

The authors were supported in part by the NSF. 
and Klainerman (see [6], Lemma 6.6.8, and also [7], [13]) that is well adapted to our approach since it only uses the Euclidean rotation and scaling vector fields and involves $1 /|x|$ decay.

The translation, rotation, and scaling vector fields are useful for obstacle problems since their normal components to the boundary of the obstacle in space-time are $O(1)$. The Lorentz boost fields, which were also used in the original generalized energy approach [9], do not have this property for any obstacle: these fields $t \partial_{i}+x_{i} \partial_{t}, i=1,2,3$, have normal components of size $t$. Consequently, it seems difficult to use these Lorentz boosts and still obtain optimal results.

In the Minkowski space (single-speed) setting all of the generators of the Lorentz group can be used without difficulty just by using the fact that they have favorable commutation properties with the D'Alembertian. In the case of an obstacle problem, however, not even the Euclidean rotation or scaling vector fields commute with the Dirichlet-wave operator. Because of the boundary conditions, the generalized energy estimates here are more involved than they are for the Minkowski space setting, particularly when these estimates involve the scaling vector field $t \partial_{t}+x \cdot \nabla_{x}$. For the scaling field we have to use our assumption that the obstacle is star-shaped in an argument that is reminiscent of that of Morawetz 18 .

We now describe more precisely the initial boundary value problems we shall consider. We assume that the obstacle $\mathcal{K} \subset \mathbb{R}^{3}$ is smooth and strictly star-shaped with respect to the origin. By this, we understand that in polar coordinates $x=r \omega$, $(r, \omega) \in[0, \infty) \times S^{2}$, we can write

$$
\mathcal{K}=\{(r, \omega): \phi(\omega)-r \geq 0\},
$$

where $\phi$ is a smooth positive function on $S^{2}$. Thus,

$$
0 \in \mathcal{K} \text {, but } 0 \notin \partial \mathcal{K}=\{x: r=\phi(\omega)\} .
$$

For such $\mathcal{K} \subset \mathbb{R}^{3}$, we consider smooth, quadratic, quasilinear systems of the form

$$
\left\{\begin{array}{l}
\square_{c} u=Q\left(d u, d^{2} u\right), \quad(t, x) \in \mathbb{R}_{+} \times \mathbb{R}^{3} \backslash \mathcal{K} \\
\left.u(t, \cdot)\right|_{\mathcal{K}}=0 \\
u(0, \cdot)=f, \partial_{t} u(0, \cdot)=g .
\end{array}\right.
$$

Here

$$
\square_{c}=\left(\square_{c_{1}}, \square_{c_{2}}, \ldots, \square_{c_{N}}\right)
$$

is a vector-valued multiple-speed D'Alembertian with

$$
\square_{c_{I}}=\partial_{t}^{2}-c_{I}^{2} \Delta
$$

where we assume that the wave speeds $c_{I}$ are all positive but not necessarily distinct. Here $\Delta=\partial_{1}^{2}+\partial_{2}^{2}+\partial_{3}^{2}$ is the standard Laplacian.

By quasilinear we mean that the nonlinear term $Q\left(d u, d^{2} u\right)$ is linear in the second derivatives of $u$. We shall also assume that the highest-order nonlinear terms are symmetric, by which we mean that, if we let $\partial_{0}=\partial_{t}$, then

$$
Q^{I}\left(d u, d^{2} u\right)=B^{I}(d u)+\sum_{\substack{0 \leq j, k, l \leq 3 \\ 1 \leq J, K \leq N}} B_{K, l}^{I J, j k} \partial_{l} u^{K} \partial_{j} \partial_{k} u^{J}, \quad 1 \leq I \leq N
$$


with $B^{I}(d u)$ a quadratic form in the gradient of $u$, and $B_{K, l}^{I J, j k}$ real constants satisfying the symmetry conditions

$$
B_{K l}^{I J, j k}=B_{K l}^{J I, j k}=B_{K l}^{I J, k j} .
$$

The second equation here places no restriction on our systems as we may obviously ensure this by symmetrizing. The first equality in (1.5) will be used when we prove the standard energy estimates. Some restriction along these lines seems necessary for our theorem to be true. In fact, there are even simple examples of linear secondorder systems that violate (1.5) and for which the basic energy estimate fails. (This failure is well known; for example, it is pointed out by Fritz John in his work on elasticity.) For completeness, we will sketch one such example following Proposition 3.2 below.

In order to solve (1.2) we must also assume that the data satisfies the relevant compatibility conditions. Since these are well known (see, e.g., [10]), we shall describe them briefly. To do so we first let $J_{k} u=\left\{\partial_{x}^{\alpha} u: 0 \leq|\alpha| \leq k\right\}$ denote the collection of all spatial derivatives of $u$ of order up to $k$. Then if $m$ is fixed and if $u$ is a formal $H^{m}$ solution of (1.2) we can write $\partial_{t}^{k} u(0, \cdot)=\psi_{k}\left(J_{k} f, J_{k-1} g\right)$, $0 \leq k \leq m$, for certain compatibility functions $\psi_{k}$ which depend on the nonlinear term $Q$ as well as $J_{k} f$ and $J_{k-1} g$. Having done this, the compatibility condition for (1.2) with $(f, g) \in H^{m} \times H^{m-1}$ is just the requirement that the $\psi_{k}$ vanish on $\partial \mathcal{K}$ when $0 \leq k \leq m-1$. Additionally, we shall say that $(f, g) \in C^{\infty}$ satisfy the compatibility conditions to infinite order if this condition holds for all $m$.

We can now state our main result. In describing the initial data we shall use the weight

$$
\langle x\rangle \equiv\left(1+|x|^{2}\right)^{\frac{1}{2}} .
$$

Theorem 1.1. Let $\mathcal{K}$ be a star-shaped obstacle, and assume that $Q\left(d u, d^{2} u\right)$ and $\square_{c}$ are as above. Assume further that $(f, g) \in C^{\infty}\left(\mathbb{R}^{3} \backslash \mathcal{K}\right)$ satisfies the compatibility conditions to infinite order.

Then there are constants $\kappa, \varepsilon_{0}>0$, and an integer $N>0$ so that for all $\varepsilon \leq \varepsilon_{0}$, if

$$
\sum_{|\alpha| \leq N}\left\|\langle x\rangle^{|\alpha|} \partial_{x}^{\alpha} f\right\|_{L^{2}\left(\mathbb{R}^{3} \backslash \mathcal{K}\right)}+\sum_{|\alpha| \leq N-1}\left\|\langle x\rangle^{|\alpha|+1} \partial_{x}^{\alpha} g\right\|_{L^{2}\left(\mathbb{R}^{3} \backslash \mathcal{K}\right)} \leq \varepsilon,
$$

then (1.2) has a unique solution $u \in C^{\infty}\left(\left[0, T_{\varepsilon}\right] \times \mathbb{R}^{3} \backslash \mathcal{K}\right)$, with

$$
T_{\varepsilon}=\exp (\kappa / \varepsilon) .
$$

The norms in which we control the solution up to time $T_{\varepsilon}$ are found in $\$ 10$.

We shall actually establish existence of limited regularity almost global solutions $u$ for data $(f, g) \in H^{N} \times H^{N-1}$ satisfying the relevant compatibility conditions. The fact that $u$ must be smooth if $f$ and $g$ are smooth and satisfy the compatibility conditions of infinite order follows from standard local existence theorems (see [10. $\S 9]$ ). Also, we are not concerned here with minimal regularity issues. The value $N=15$, which we eventually require (see (10.1) below), is certainly not optimal.

Together with the finite propagation speed of our equations, the blow-up examples in, e.g., John [8] show that for the class of nonlinearities described above, the time of existence (1.8) is sharp. If we restrict our attention to null-form nonlinearities and single-speed systems, global-in-time solutions outside of star-shaped obstacles were established by the authors in [10]. This extended earlier spherically 
symmetric work of 4]. For related work outside of obstacles in higher dimensions see [5], [28].

We point out that results similar to those in Theorem 1.1 were announced in Datti [2], but there appears to be a gap in the argument which has not been repaired. Specifically, the proof of Theorem 5.3 of [2] cannot be attained as claimed, and hence the main estimates of the paper remain unproven.

As we remarked before, we can also give a proof of a multiple-speed generalization of the almost global existence theorem of John and Klainerman [9]:

Theorem 1.2. Assume that $Q\left(d u, d^{2} u\right)$ and $\square_{c}$ are as above. Then there exists $N>0$ and constants $\kappa, \varepsilon_{0}>0$ so that for all $\varepsilon<\varepsilon_{0}$ and data $(f, g) \in C^{\infty}\left(\mathbb{R}^{3}\right) \cap$ $L^{6}\left(\mathbb{R}^{3}\right)$ satisfying

$$
\sum_{|\alpha| \leq N}\left\|\langle x\rangle^{|\alpha|} \partial_{x}^{\alpha} f^{\prime}\right\|_{L^{2}\left(\mathbb{R}^{3}\right)}+\sum_{|\alpha| \leq N-1}\left\|\langle x\rangle^{|\alpha|} \partial_{x}^{\alpha} g\right\|_{L^{2}\left(\mathbb{R}^{3}\right)} \leq \varepsilon,
$$

the system

$$
\left\{\begin{array}{l}
\square_{c} u=Q\left(d u, d^{2} u\right), \\
u(0, \cdot)=f, \partial_{t} u(0, \cdot)=g
\end{array}\right.
$$

has a unique solution $u \in C^{\infty}\left(\left[0, T_{\varepsilon}\right] \times \mathbb{R}^{3}\right)$, where

$$
T_{\varepsilon}=\exp (\kappa / \varepsilon) .
$$

As we noted earlier, in [14] Klainerman and Sideris established Theorem 1.2 in the case of certain divergence-form nonlinearities without using Lorentz boost vector fields. Also, it seems clear that the techniques of Sideris [22] can handle the special case of Theorem 1.2 where the semilinear terms $B^{I}(d u)$ are not present.

We eventually choose $N=10$ in Theorem 1.2 (see (3.12) below). The decay we obtain up until time $T_{\varepsilon}$ is described in equations (3.15) -3.16) below.

Global existence in three space dimensions has been shown for coupled multiplespeed systems satisfying various multiple-speed versions of the so-called null condition [13]. See Sideris and Tu 24], Sogge [27] for such global, multiple-speed results and further references. These results generalize the first global existence results of Christodoulou [1] and Klainerman [13. Long-time existence for multiple-speed systems in two space dimensions was studied in Kovalyov [15].

This paper is organized as follows. In the next section we shall prove some new pointwise $L^{1} \rightarrow L^{\infty}$ estimates for the inhomogeneous wave equation in Minkowski space that are well adapted to our approach of trying to mainly exploit $1 /|x|$ decay of solutions of nonlinear wave equations. From the point of view of the Minkowski space argument of Theorem [1.2] this estimate is a departure from the approach of Klainerman and Sideris [14. After this, we recall the weighted space-time $L^{2}$ estimates from [11] and give the straightforward iteration argument which proves Theorem 1.2. We then turn to the obstacle case, obtaining versions of the pointwise decay, weighted space-time $L^{2}$ estimates, and fixed-time $L^{2}$ estimates in the exterior of a star-shaped obstacle. As pointed out above, the energy estimates for the boundary value problem are more involved than their Minkowski space analogues, and in fact our estimates involving the Euclidean rotation or scaling vector fields involve a slight loss over their Minkowski variants. Fortunately this loss is not important for our goal of proving Theorem 1.1 Finally, in \$10, we combine the 
decay, weighted $L^{2}\left(\mathbb{R}^{1+3}\right)$, and energy estimates outside of obstacles in an adaptation of the proof of Theorem 1.2 to obtain our almost global existence results for quasilinear wave equations outside of star-shaped obstacles.

\section{Pointwise estimates in Minkowski space}

We write $\{\Omega\}=\left\{\Omega_{i j}\right\}$, where

$$
\Omega_{i j}=x_{i} \partial_{j}-x_{j} \partial_{i}, \quad 1 \leq i<j \leq 3,
$$

are the Euclidean $\mathbb{R}^{3}$ rotation operators. Denote by $Z$ either a space-time translation or spatial rotation vector field,

$$
\{Z\}=\left\{\partial_{t}, \partial_{j}, \Omega_{i j}\right\}
$$

We also use the scaling operator

$$
L=t \partial_{t}+x \cdot \nabla_{x}=t \partial_{t}+r \partial_{r} .
$$

Throughout the remainder of the paper we will use without explicit mention the following fact: if we denote by $\Gamma$ any of the vector fields in (2.2)- 2.3 , then

$$
\left[\Gamma_{i}, \Gamma_{j}\right]=\sum_{k} \mu_{i j k} \Gamma_{k}
$$

for certain (possibly vanishing) fixed constants $\mu_{i j k}$.

To simplify the notation, we let

$$
\square=\partial_{t}^{2}-\Delta
$$

be the scalar unit-speed D'Alembertian. We shall state most of our estimates in terms of it, rather than the multiple-speed operator $\square_{c}$ in (1.3) since straightforward scaling arguments will show that our estimates for $\square$ yield ones for $\square_{c}$.

Having set up the notation, we can now state one of our main results, which is the following variant of an estimate of John, Klainerman, and Hörmander ([ 6 , Lemma 6.6.8).

Proposition 2.1. If $w \in C^{5}$ and $\square w=F$ in $[0, t] \times \mathbb{R}^{3}$, and the Cauchy data of $w$ vanishes at $t=0$, then

$$
(1+t)|w(t, x)| \leq C \int_{0}^{t} \int_{\mathbb{R}^{3}} \sum_{|\alpha|+j \leq 3, j \leq 1}\left|L^{j} Z^{\alpha} F(s, y)\right| \frac{d y d s}{|y|} .
$$

To prove this estimate we use the following.

Lemma 2.2. Let $w$ be as above, and fix $x \in \mathbb{R}^{3}$ with $|x|=r$. Then,

$$
|x||w(t, x)| \leq \frac{1}{2} \int_{0}^{t} \int_{|r-(t-s)||\theta|=1}^{r+t-s} \sup _{|\theta|}|F(s, \rho \theta)| \rho d \rho d s .
$$

Proof. (Lemma 2.2). This result is well known (see, e.g., p. 8 of Sogge [26]). Since the fundamental solution of the wave equation in $1+3$ dimensions is positive, we have that $|w| \leq|W|$, where $W$ is the solution of the inhomogeneous wave equation $\square W(t, y)=G(t,|y|)$ and $G$ is the radial majorant of $F$,

$$
G(t, \rho)=\sup _{\theta \in S^{2}}|F(t, \rho \theta)| .
$$


On the other hand, $W(t, y)$ is a spherically symmetric solution to the wave equation in three space dimensions. Hence $|y| W(t, y)$ satisfies the wave equation in one space dimension with forcing term $|y| G(t,|y|)$,

$$
|x| W(t, x)=\frac{1}{2} \int_{0}^{t} \int_{|r-(t-s)|}^{r+t-s} G(s, \rho) \rho d \rho d s .
$$

Together, (2.6), (2.7) yield (2.5).

Proof. (Proposition 2.1): As in [6], we first prove the following:

$$
t|w(t, x)| \leq C \int_{0}^{t} \int_{\mathbb{R}^{3}} \sum_{|\alpha| \leq 2, j \leq 1}\left|L^{j} \Omega^{\alpha} F(s, y)\right| \frac{d y d s}{|y|} .
$$

Since the estimate (2.8) is scale invariant, it suffices by scaling to prove the bounds for $t=1$, that is,

$$
|w(1, x)| \leq C \int_{0}^{1} \int_{\mathbb{R}^{3}} \sum_{|\alpha| \leq 2, j \leq 1}\left|L^{j} \Omega^{\alpha} F(s, y)\right| \frac{d y d s}{|y|} .
$$

Let us first prove the estimate for those $|x|>1 / 10$. By the Sobolev Lemma,

$$
\sup _{|\theta|=1}|F(s, \rho \theta)| \leq C \sum_{|\alpha| \leq 2} \int_{S^{2}}\left|\left(\Omega^{\alpha} F\right)(s, \rho \theta)\right| d \theta .
$$

Together with (2.5) this gives

$$
|x||w(1, x)| \leq C \sum_{|\alpha| \leq 2} \int_{0}^{1} \int_{\mathbb{R}^{3}}\left|\Omega^{\alpha} F(s, y)\right| \frac{d y d s}{|y|},
$$

which proves (2.9) when $|x|>1 / 10$.

It remains to consider (2.9) for a fixed $|x| \leq 1 / 10$. Since the estimate (2.9) only involves homogeneous derivatives, and hence is preserved under cutoffs of the form $\psi(y /|x|)$, with $\psi$ a radial bump function, we can reduce matters to considering two cases:

- Case 1: $\operatorname{supp} F \subset\{(s, y):|y| \geq 2|x|\}$,

- Case 2: $\operatorname{supp} F \subset\{(s, y):|y| \leq 4|x|\}$.

For both cases we use the formula for $w$ coming from the fundamental solution,

$$
w(t, x)=\frac{1}{4 \pi} \int_{|y|<t} F(t-|y|, x-y) \frac{d y}{|y|} .
$$

Case 1: In this case $F(s, x-y)=0$ for $|y| \leq|x|$. Hence

$$
|w(1, x)| \leq \int_{|y|<1}|F(1-|y|, x-y)| \frac{d y}{|x-y|} .
$$

Note that $|(1-|y|, x-y)| \geq 1 / 4$ on the support of the integrand. Thus, if $\rho(s) \in$ $C^{\infty}(\mathbb{R})$ vanishes for $s<1 / 8$ and equals one for $s>1 / 4$ we have

$$
|w(1, x)| \leq \int_{|y|<1} H(1-|y|, x-y) d y,
$$

where

$$
H(s, v)=\rho(|(s, v)|)|F(s, v)| /|v| .
$$


We make the change of variables $\varphi(\tau, y)=\tau(1-|y|, x-y)$, where $|y| \leq 1$ and $0<\tau<1$. The Jacobian is $\tau^{3}(\langle x, y\rangle /|y|-1)$. It is bounded away from zero when $H(\varphi(\tau, y)) \neq 0$ since we are assuming that $|x|<1 / 10$, and since $H(s, v)=0$ when $|(s, v)|<1 / 8$. Also,

$$
\begin{aligned}
\int_{|y|<1} H(1-|y|, x-y) d y & =\int_{|y|<1} \mid H(\varphi(1, y) \mid d y \\
& \leq C \iint_{|y|<1,0<\tau<1}\left(\mid H\left(\varphi(\tau, y)|+| \frac{\partial}{\partial \tau} H(\varphi(\tau, y) \mid) d \tau d y\right.\right.
\end{aligned}
$$

Note that $|\partial H(\varphi(\tau, y)) / \partial \tau|=\mid(L H)(\varphi(\tau, y) \mid / \tau$, and since $\tau$ is bounded from below when $H(\varphi(\tau, y)) \neq 0$, we conclude that

$$
\begin{aligned}
|w(1, x)| & \leq C \iint_{0<s<1}(|H(s, y)|+|L H(s, y)|) d y d s \\
& \leq C \iint_{0<s<1}(|F(s, y)|+|L F(s, y)|) \frac{d y d s}{|y|},
\end{aligned}
$$

as desired.

Case 2: Our assumptions here are $F(s, y)=0$ when $|y| \geq 4|x|$, for some fixed $x$ with $|x|<1 / 10$. In this case, we have $w(1, x)=w_{0}(1, x)$ where $w_{0}$ solves the inhomogeneous wave equation $\square w_{0}(t, y)=G(t, y)$, with $G(t, y)=F(t, y)$ if $t \geq 1-5|x|$, and $G(t, y)=0$ otherwise. By (2.10),

$$
\begin{aligned}
|w(1, x)|=\left|w_{0}(1, x)\right| & \leq \frac{C}{|x|} \int_{1-5|x|}^{1} \int \sum_{|\alpha| \leq 2}\left|\Omega^{\alpha} F(s, y)\right| \frac{d s d y}{|y|} \\
& \leq C \sup _{1 / 2<s<1} \int \sum_{|\alpha| \leq 2}\left|\Omega^{\alpha} F(s, y)\right| \frac{d y}{|y|} .
\end{aligned}
$$

As in Case 1, we bound this last quantity using the fundamental theorem of calculus,

$$
\begin{aligned}
F(s, y) & =\int_{0}^{1} \frac{d}{d \tau} G(\tau s, \tau y) d \tau \\
& =\int_{0}^{1} L G(\tau s, \tau y) d \tau, \quad \frac{1}{2} \leq s \leq 1 .
\end{aligned}
$$

Hence we have

$$
\begin{aligned}
|w(1, x)| & \leq C \sup _{1 / 2<s<1} \int_{0}^{1} \int \sum_{|\alpha| \leq 2}\left|\Omega^{\alpha} L G(\tau s, \tau y)\right| d \tau \frac{d y}{|y|} \\
& \leq C \int_{0}^{1} \int \sum_{|\alpha| \leq 2}\left|\Omega^{\alpha} L F(s, y)\right| \frac{d y}{|y|}
\end{aligned}
$$

where, similar to Case 1 above, we have used the fact that $\left|\frac{\partial(\tau s, \tau y)}{\partial(\tau, y)}\right|^{-1}$ is bounded on the support of $G$. This completes the discussion of case 2 .

Exactly as in [6], the desired bound (2.4) follows from (2.8). More precisely, if supp $F \subset\{(s, y): s \geq 1\}$, then the same is true for supp $w$, and (2.4) follows immediately from (2.8). In case supp $F \subset\{(s, y): 0 \leq s \leq 1\}$, then we apply the previous argument to the function $\tilde{w}$ with $\square \tilde{w}=F\left(s-2, y_{1}, y_{2}, y_{3}\right)$. The translation 
introduces the usual Euclidean derivatives and gives (2.4) by the preceding argument. The case of a general forcing function $F$ follows from these considerations and a partition of unity.

\section{3. $L_{x}^{2}, L_{x, t}^{2}$ ESTIMATES AND ALMOST GLOBAL EXISTENCE FOR QUASILINEAR EQUATIONS IN MINKOWSKI SPACE}

We now use the pointwise estimates in Proposition 2.1 along with $L_{t}^{2} L_{x}^{2}$ estimates exploiting $1 / r$ decay of solutions of the wave equation to prove Theorem 1.2 the almost global existence theorem for certain multiple-speed systems. As we shall see, this proof provides a simple model for the proof of almost global existence results in the presence of obstacles.

To do this we need to use a simple modification of an estimate from [11] which involves the scalar D'Alembertian $\square=\partial_{t}^{2}-\Delta$.

Proposition 3.1. Suppose that $v$ solves the wave equation $\square v=G$ on $\mathbb{R}_{+} \times \mathbb{R}^{3}$, with Cauchy data $f \in \dot{H}^{1} \cap L^{6}\left(\mathbb{R}^{3}\right), g \in L^{2}\left(\mathbb{R}^{3}\right)$ at $t=0$. Then there is a constant $C$ so that

$$
\begin{aligned}
& (\ln (2+t))^{-1 / 2}\left\|\langle x\rangle^{-1 / 2} v^{\prime}\right\|_{L^{2}\left([0, t] \times \mathbb{R}^{3}\right)}+\left\|\langle x\rangle^{-1} v\right\|_{L_{s}^{2} L_{x}^{6}\left([0, t] \times \mathbb{R}^{3}\right)} \\
& \leq C\|(f, g)\|_{\dot{H}^{1} \times L^{2}\left(\mathbb{R}^{3}\right)}+C \int_{0}^{t}\|G(s, \cdot)\|_{L^{2}\left(\mathbb{R}^{3}\right)} d s .
\end{aligned}
$$

Here, and in what follows, $v^{\prime}$ denotes the space-time gradient of $v$, i.e., $v^{\prime}=$ $\left(\partial_{t} v, \nabla_{x} v\right)$.

We sketch the proof of (3.1); more details appear in [11]. The bound is achieved by considering separately two regions of $\{(s, x): 0 \leq s \leq t\}$. Specifically, if the norms on the left of (3.1) are taken over $\{(s, x): 0 \leq s \leq t,|x| \geq t\}$, the estimate follows immediately from

$$
\begin{aligned}
& \langle t\rangle^{-1 / 2}\left(\left\|v^{\prime}\right\|_{L^{2}\left([0, t] \times \mathbb{R}^{3}\right)}+\|v\|_{L_{s}^{2} L_{x}^{6}\left([0, t] \times \mathbb{R}^{3}\right)}\right) \\
& \leq\|(f, g)\|_{\dot{H}^{1} \times L^{2}\left(\mathbb{R}^{3}\right)}+\int_{0}^{t}\|G(s, \cdot)\|_{L^{2}\left(\mathbb{R}^{3}\right)} d s,
\end{aligned}
$$

which is in turn an immediate consequence of the standard fixed-time energy estimate and Sobolev embedding. We remark that the condition $f \in L^{6}$ implies that $f$, hence $v(s, \cdot)$ for all $s$, is the $\dot{H}^{1}$ limit of compactly supported functions, which allows us to bound $\|v(s, \cdot)\|_{L^{6}} \leq C\left\|v^{\prime}(s, \cdot)\right\|_{L^{2}}$.

To establish (3.1) on the region $\{(s, x): 0 \leq s \leq t,|x|<t\}$, we first show that

$$
\begin{aligned}
& \left\|v^{\prime}\right\|_{L^{2}([0, t] \times\{|x|<1\})}+\|v\|_{L_{s}^{2} L_{x}^{6}([0, t] \times\{|x|<1\})} \\
& \quad \leq C\|(f, g)\|_{\dot{H}^{1} \times L^{2}\left(\mathbb{R}^{3}\right)}+C \int_{0}^{t}\|G(s, \cdot)\|_{L^{2}\left(\mathbb{R}^{3}\right)} d s .
\end{aligned}
$$

For the term involving $v^{\prime}$ on the left, this can be shown using the energy inequality and the sharp Huygens principle (see [11] for details1). To handle the term in $v$, we note that by the Duhamel principal we may take $G=0$. By Sobolev embedding, we can reduce matters to showing that

$$
\|v\|_{L^{2}(\mathbb{R} \times\{|x|<1\})} \leq C\|(f, g)\|_{\dot{H}^{1} \times L^{2}\left(\mathbb{R}^{3}\right)} .
$$

\footnotetext{
[20].

${ }^{1}$ In fact, the bound (3.2) is also implicit in several previous works, going back at least to [19],
} 
To verify this last estimate, we let $\chi(x)$ denote the cutoff to the set $|x|<1$. Then by the Plancherel theorem, we have

$$
\begin{aligned}
\|\chi v\|_{L_{t}^{2} L_{x}^{2}\left(\mathbb{R} \times \mathbb{R}^{3}\right)} & =\|(\hat{\chi} * \hat{v})(\tau, \xi)\|_{L_{\tau}^{2} L_{\xi}^{2}\left(\mathbb{R} \times \mathbb{R}^{3}\right)} \\
& \leq C\|\hat{v}(\tau, \xi)\|_{L_{\tau}^{2} L_{\xi}^{1}} \leq C\|(f, g)\|_{\dot{H}^{1} \times L^{2}\left(\mathbb{R}^{3}\right)},
\end{aligned}
$$

where the last inequality is seen by expressing $\hat{v}$ in terms of $(\hat{f}, \hat{g})$, and representing the $\xi$ integral in polar coordinates. Applying the Schwarz inequality to the angular integral yields the desired bound.

A scaling argument applied to (B.2) yields

$$
\begin{aligned}
&\left\|\langle x\rangle^{-1 / 2} v^{\prime}\right\|_{L^{2}([0, t] \times\{R<|x|<2 R\})}+\left\|\langle x\rangle^{-1 / 2} v\right\|_{L_{s}^{2} L_{x}^{6}([0, t] \times\{R<|x|<2 R\})} \\
& \leq C\|(f, g)\|_{\dot{H}^{1} \times L^{2}\left(\mathbb{R}^{3}\right)}+C \int_{0}^{t}\|G(s, \cdot)\|_{L^{2}\left(\mathbb{R}^{3}\right)} d s .
\end{aligned}
$$

The estimate for the first term in the left side of (3.1) on $\{|x| \leq t\}$ now follows by squaring the left-hand side, decomposing dyadically in $r$, using (3.3) for each piece, and adding the resulting estimates. One estimates the second term in the left side of (3.1) using (3.3) and the fact that this second term involves the weight $\langle x\rangle^{-1}$. The extra weight of $\langle x\rangle^{-\frac{1}{2}}$ allows us to sum the estimates for the dyadic pieces with no growth in $t$.

In addition to this estimate we shall also need the standard energy estimate:

Proposition 3.2. Let $\gamma^{I J, i j}(t, x), 1 \leq I, J \leq N, 0 \leq i, j \leq 3$ be real $C^{0,1}$ functions satisfying

$$
\sum_{0 \leq I, J \leq N} \sum_{0 \leq i, j \leq 3}\left|\gamma^{I J, i j}\right|<\frac{1}{2} \min \left(c_{I}^{2}\right), \quad 1 \leq I \leq N, 0 \leq t \leq T
$$

as well as

$$
\int_{0}^{T} \sum_{0 \leq I, J \leq N} \sum_{0 \leq i, j \leq 3}\left\|\nabla_{t, x} \gamma^{I J, i j}(t, \cdot)\right\|_{L^{\infty}\left(\mathbb{R}^{3}\right)} d t<1 .
$$

Assume also that $\gamma^{I J, i j}$ satisfies the symmetry condition

$$
\gamma^{I J, i j}=\gamma^{J I, i j}=\gamma^{I J, j i} \text {. }
$$

Then if

$$
\left(\partial_{t}^{2}-c_{I}^{2} \Delta\right) v^{I}=\sum_{J=1}^{N} \sum_{0 \leq i, j \leq 3} \gamma^{I J, i j} \partial_{i} \partial_{j} v^{J}+F^{I}, \quad 1 \leq I \leq N
$$

there is a constant $C$, independent of $\gamma^{I J, i j}, F$, and $T$, so that

$$
\left\|v^{\prime}(t, \cdot)\right\|_{L^{2}\left(\mathbb{R}^{3}\right)} \leq C\left\|v^{\prime}(0, \cdot)\right\|_{L^{2}\left(\mathbb{R}^{3}\right)}+C \int_{0}^{t}\|F(s, \cdot)\|_{L^{2}\left(\mathbb{R}^{3}\right)} d s, \quad 0 \leq t \leq T .
$$

We omit the standard proof of (3.7), since analogous estimates for Dirichlet-wave equations will be proven in $\S 5$. We observe here, though, that the energy estimate can fail in the absence of the symmetry assumption (3.6). To see this, consider the following nonsymmetric linear homogeneous system on $\mathbb{R} \times \mathbb{R}^{3}$ :

$$
\begin{aligned}
& \square u=0, \\
& \square v=\frac{1}{4} \partial_{t}^{2} u,
\end{aligned}
$$


with $u, v, \partial_{t} v$ all vanishing at $t=0$, and with $\partial_{t} u(0, \cdot)=g$.

Then $\left\|\left(u^{\prime}, v^{\prime}\right)(0, \cdot)\right\|_{L^{2}\left(\mathbb{R}^{3}\right)}=\|g\|_{L^{2}\left(\mathbb{R}^{3}\right)}$, and the standard energy estimate shows that

$$
\left\|\left(u^{\prime}, v^{\prime}\right)(t, \cdot)\right\|_{L^{2}\left(\mathbb{R}^{3}\right)}^{2}=\int_{\mathbb{R}^{3}}|g(x)|^{2} d x+\frac{1}{2} \int_{0}^{t} \int_{\mathbb{R}^{3}} v_{s}(s, x) u_{s s}(s, x) d x d s .
$$

Using the Fourier transform and Duhamel's principle, it is straightforward to see that the second term on the right-hand side of (3.8) is comparable to $\left\|g^{\prime}\right\|_{L^{2}\left(\mathbb{R}^{3}\right)}^{2}$.

We shall actually require a corollary to Proposition 3.2 which is based on the following commutator relations $\left[\left(\partial_{t}^{2}-c_{I}^{2} \Delta\right), Z\right]=0$ (see (2.2) $)$ and $\left[\left(\partial_{t}^{2}-c_{I}^{2} \Delta\right), L\right]=$ $2\left(\partial_{t}^{2}-c_{I}^{2} \Delta\right)$, where, as above, $L$ is the scaling vector field (2.3).

Corollary 3.3. Let $\gamma^{I J, i j}(t, x) \in C^{\infty}$ satisfy (3.4) $-(\underline{3.6)}$, and let $v$ and $F$ be as in Proposition 3.2. Then if $M=1,2, \ldots$ is fixed there is a constant $C$, independent of $\gamma^{I J, i j}, F$, and $T$, so that for $0 \leq t \leq T$,

$$
\begin{aligned}
& \sum_{|\alpha|+m \leq M}\left\|L^{m} Z^{\alpha} v^{\prime}(t, \cdot)\right\|_{L^{2}\left(\mathbb{R}^{3}\right)} \\
& \leq C \sum_{|\alpha|+m \leq M}\left\|L^{m} Z^{\alpha} v^{\prime}(0, \cdot)\right\|_{L^{2}\left(\mathbb{R}^{3}\right)} \\
&+C \int_{0}^{t} \sum_{\substack{|\alpha|+m \leq M\\
}}\left\|L^{m} Z^{\alpha} F(s, \cdot)\right\|_{L^{2}\left(\mathbb{R}^{3}\right)} d s \\
&+C \int_{0}^{t} \sum_{\substack{|\alpha|+m \leq M \\
I, J, i, j}}\left\|\left[L^{m} Z^{\alpha}, \gamma^{I J, i j} \partial_{i} \partial_{j}\right] v^{J}(s, \cdot)\right\|_{L^{2}\left(\mathbb{R}^{3}\right)} d s
\end{aligned}
$$

We note that if we restrict $m \leq 1$ on the left-hand side, then we may take $m \leq 1$ on the right-hand side as well. We shall also need the following consequence of the Sobolev lemma, see Klainerman [12]:

Lemma 3.4. Suppose that $h \in C^{\infty}\left(\mathbb{R}^{3}\right)$. Then for $R>1$,

$$
\|h\|_{L^{\infty}(R / 2<|x|<R)} \leq C R^{-1} \sum_{|\alpha|+|\gamma| \leq 2}\left\|\Omega^{\alpha} \partial_{x}^{\gamma} h\right\|_{L^{2}(R / 4<|x|<2 R)} .
$$

To handle certain higher-order commutator terms that arise in our arguments, we will also use the following variant of an estimate of Klainerman and Sideris (see [14], Lemma 3.1).

Lemma 3.5. Suppose that $1 \leq R \leq c t / 4$. Then for $0 \leq j \leq 3$,

$$
\begin{gathered}
\left\|\partial_{j} v^{\prime}(t, \cdot)\right\|_{L^{2}(R / 2<|x|<R)} \leq C(1+t)^{-1} \sum_{|\alpha|+m \leq 1}\left\|L^{m} Z^{\alpha} v^{\prime}(t, \cdot)\right\|_{L^{2}(R / 4<|x|<2 R)} \\
+C R^{-1}\left(\left\|v^{\prime}(t, \cdot)\right\|_{L^{2}(R / 4<|x|<2 R)}+\|v(t, \cdot)\|_{L^{6}(R / 4<|x|<2 R)}\right) \\
+C\left\|\left(\partial_{t}^{2}-c^{2} \Delta\right) v\right\|_{L^{2}(R / 4<|x|<2 R)} .
\end{gathered}
$$


Also,

$$
\begin{aligned}
\left\|\partial_{j} v^{\prime}(t, \cdot)\right\|_{L^{2}(|x|<1)} & \leq C(1+t)^{-1} \sum_{|\alpha|+m \leq 1}\left\|L^{m} Z^{\alpha} v^{\prime}(t, \cdot)\right\|_{L^{2}\left(\mathbb{R}^{3}\right)} \\
& +C\left(\left\|v^{\prime}(t, \cdot)\right\|_{L^{2}(|x|<2)}+\|v(t, \cdot)\|_{L^{6}(|x|<2)}\right) \\
& +C\left\|\left(\partial_{t}^{2}-c^{2} \Delta\right) v\right\|_{L^{2}(|x|<2)} .
\end{aligned}
$$

The constant $C$ depends only on $c$.

Proof. By scaling we may take the wave speed $c$ to be one. We then use the fact (see 14], Lemma 2.3) that for $|x|<t / 2$,

$$
\left|\partial_{t} v^{\prime}(t, x)\right|+|\Delta v(t, x)| \leq C(1+t)^{-1} \sum_{|\alpha|+m \leq 1}\left|L^{m} Z^{\alpha} v^{\prime}(t, x)\right|+C\left|\left(\partial_{t}^{2}-\Delta\right) v(t, x)\right| .
$$

Using this we immediately get the estimates for $j=0$. The other cases of (3.10) follow from the $j=0$ bound and the fact that, for $j, k=1,2,3$,

$$
\begin{aligned}
& \left\|\partial_{j} \partial_{k} v(t, \cdot)\right\|_{L^{2}(R / 2<|x|<R)} \\
& \leq C\|\Delta v(t, \cdot)\|_{L^{2}(R / 4<|x|<2 R)}+C \sum_{|\alpha| \leq 1} R^{-2+|\alpha|}\left\|\partial_{x}^{\alpha} v(t, \cdot)\right\|_{L^{2}(R / 4<|x|<2 R)} \\
& \leq C\|\Delta v(t, \cdot)\|_{L^{2}(R / 4<|x|<2 R)} \\
& \quad+C R^{-1}\left(\left\|v^{\prime}(t, \cdot)\right\|_{L^{2}(R / 4<|x|<2 R)}+\|v\|_{L^{6}(R / 4<|x|<2 R)}\right) .
\end{aligned}
$$

The inequality (3.11) follows by a similar argument.

We now use Propositions 2.1 and 3.1 along with Corollary 3.3 to prove Theorem 1.2. We are assuming that the data $f, g \in C^{\infty}\left(\mathbb{R}^{3}\right) \cap L^{6}\left(\mathbb{R}^{3}\right)$ satisfy the smallness condition

$$
\sum_{|\alpha| \leq 10}\left\|\langle x\rangle^{|\alpha|} \partial_{x}^{\alpha} f^{\prime}\right\|_{L^{2}\left(\mathbb{R}^{3}\right)}+\sum_{|\alpha| \leq 10}\left\|\langle x\rangle^{|\alpha|} \partial_{x}^{\alpha} g\right\|_{L^{2}\left(\mathbb{R}^{3}\right)} \leq \varepsilon,
$$

where $\varepsilon>0$ is small and we aim to show that there is a solution on $\left[0, T_{\varepsilon}\right] \times \mathbb{R}^{3}$, verifying

$$
\begin{array}{r}
\sup _{0 \leq t \leq T_{\varepsilon}}\left(\sum_{|\alpha|+m \leq 10, m \leq 1}\left\|L^{m} Z^{\alpha} u^{\prime}(t, \cdot)\right\|_{L^{2}\left(\mathbb{R}^{3}\right)}+(1+t) \sum_{|\alpha| \leq 1}\left\|Z^{\alpha} u^{\prime}(t, \cdot)\right\|_{L^{\infty}\left(\mathbb{R}^{3}\right)}\right) \\
+\left(\ln \left(2+T_{\varepsilon}\right)\right)^{-1 / 2} \sum_{|\alpha|+m \leq 9, m \leq 1}\left\|\langle x\rangle^{-\frac{1}{2}} L^{m} Z^{\alpha} u^{\prime}\right\|_{L^{2}\left(\left[0, T_{\varepsilon}\right] \times \mathbb{R}^{3}\right)} \leq C \varepsilon,
\end{array}
$$

where $T_{\varepsilon}=\exp (\kappa / \varepsilon)$, with $\kappa>0$ being a uniform constant. If the initial data is $C^{\infty}$, and the solution satisfies (3.13), then standard local existence theory shows that the solution is actually $C^{\infty}$ on $\left[0, T_{\varepsilon}\right] \times \mathbb{R}^{3}$.

Set $u_{-1}=0$, and define $u_{k}, k=0,1,2, \ldots$ inductively by letting $u_{k}$ solve

$$
\begin{cases}\square_{c_{I}} u_{k}^{I}(t, x)=B^{I}\left(u_{k-1}^{\prime}\right)+\sum_{\substack{0 \leq i, j, l \leq 3 \\ 1 \leq J, K \leq N}} B_{K l}^{I J, i j} \partial_{l} u_{k-1}^{K} \partial_{i} \partial_{j} u_{k}^{J}, \\ u_{k}(0, \cdot)=f, \quad \partial_{t} u_{k}(0, \cdot)=g, & (t, x) \in\left[0, T_{\varepsilon}\right] \times \mathbb{R}^{3}, 1 \leq I \leq N\end{cases}
$$


where $\square_{c}$ is as in (1.3). Let

$$
\begin{aligned}
M_{k}(T)= & \sum_{|\alpha|+m \leq 9, m \leq 1}\left[(\ln (2+T))^{-1 / 2}\left\|\langle x\rangle^{-1 / 2} L^{m} Z^{\alpha} u_{k}^{\prime}\right\|_{L^{2}\left([0, T] \times \mathbb{R}^{3}\right)}\right. \\
& \left.+\left\|\langle x\rangle^{-1} L^{m} Z^{\alpha} u_{k}\right\|_{L_{t}^{2} L_{x}^{6}\left([0, T] \times \mathbb{R}^{3}\right)}\right] \\
& +\sup _{0 \leq t \leq T} \sum_{|\alpha|+m \leq 10, m \leq 1}\left\|L^{m} Z^{\alpha} u_{k}^{\prime}(t, \cdot)\right\|_{L^{2}\left(\mathbb{R}^{3}\right)} \\
& +\sup _{0 \leq t \leq T}(1+t) \sum_{|\alpha| \leq 1}\left\|Z^{\alpha} u_{k}^{\prime}(t, \cdot)\right\|_{L^{\infty}\left(\mathbb{R}^{3}\right)} \\
& =I_{k}(T)+I I_{k}(T)+I I I_{k}(T) .
\end{aligned}
$$

We first observe that there is a uniform constant $C_{0}$ so that

$$
M_{0}(T) \leq C_{0} \varepsilon
$$

for all $T$. This follows from the results of section 2 and the earlier $L^{2}$ estimates of this section, together with an application of the generalized Sobolev inequalities of Klainerman to obtain the pointwise decay estimates.

We claim that if $\varepsilon<\varepsilon_{0}$ is sufficiently small and if the constant $\kappa$ occurring in the definition of $T_{\varepsilon}$ is small enough, then there is a uniform constant $C$ (which will be allowed to change from line to line throughout this paper) so that for all $k=1,2,3, \ldots$,

$$
M_{k}\left(T_{\varepsilon}\right) \leq C \varepsilon
$$

We prove this inductively. We thus assume that the bound holds for $k-1$ and then establish it for $k$.

We begin by applying Corollary 3.3 , with $F=B\left(u_{k-1}^{\prime}\right)$ and

$$
\gamma^{I J, i j}=\gamma^{I J, i j}\left(u_{k-1}^{\prime}\right)=\sum_{l, K} B_{K l}^{I J, i j} \partial_{l} u_{k-1}^{K},
$$

to estimate $I I_{k}(T)$. Note that the hypotheses (3.4) and (3.5) on the metric perturbation are satisfied by the induction hypothesis if $\varepsilon$ is small and $T<T_{\varepsilon}$. The symmetry hypothesis (3.6) is also valid in view of our symmetry assumption (1.5) on the quasilinear terms. We next apply Proposition 3.1 with $G=\square_{c} L^{m} Z^{\alpha} u_{k}$ to estimate $I_{k}(T)$. We conclude that

$$
\begin{aligned}
& I_{k}+I I_{k} \leq C_{0} \varepsilon+C \int_{0}^{T_{\varepsilon}} \sum_{\substack{|\alpha|+m \leq 10 \\
m \leq 1}}\left\|L^{m} Z^{\alpha} B\left(u_{k-1}^{\prime}\right)(s, \cdot)\right\|_{L^{2}\left(\mathbb{R}^{3}\right)} d s \\
&+C \int_{0}^{T_{\varepsilon}} \sum_{\substack{|\alpha|+m \leq 9 \\
m \leq 1}}\left\|L^{m} Z^{\alpha} \square_{c} u_{k}(s, \cdot)\right\|_{L^{2}\left(\mathbb{R}^{3}\right)} d s \\
&+C \int_{0}^{T_{\varepsilon}} \sum_{I, J, i, j} \sum_{\substack{|\alpha|+m \leq 10 \\
m \leq 1}}\left\|\left[L^{m} Z^{\alpha}, \gamma^{I J, i j}\left(u_{k-1}^{\prime}\right) \partial_{i} \partial_{j}\right] u_{k}^{J}(s, \cdot)\right\|_{L^{2}\left(\mathbb{R}^{3}\right)} d s .
\end{aligned}
$$


We estimate the first integral by observing that

$$
\begin{array}{r}
\sum_{\substack{|\alpha|+m \leq 10 \\
m \leq 1}}\left|L^{m} Z^{\alpha} B\left(u_{k-1}^{\prime}\right)\right| \leq C \sum_{\substack{|\alpha|+m \leq 9 \\
m \leq 1}}\left|L^{m} Z^{\alpha} u_{k-1}^{\prime}\right| \sum_{\substack{|\alpha|+m \leq 5 \\
m \leq 1}}\left|L^{m} Z^{\alpha} u_{k-1}^{\prime}\right| \\
+C\left|u_{k-1}^{\prime}\right| \sum_{\substack{|\alpha|+m \leq 10 \\
m \leq 1}}\left|L^{m} Z^{\alpha} u_{k-1}^{\prime}\right| .
\end{array}
$$

We control the contribution of the second term on the right-hand side of (3.18) to (3.17) using the induction hypothesis (3.16) as follows:

$$
\begin{array}{r}
\int_{0}^{T_{\varepsilon}}\left\|u_{k-1}^{\prime}(s, \cdot)\right\|_{L^{\infty}\left(\mathbb{R}^{3}\right)} \sum_{\substack{|\alpha|+m \leq 10 \\
m \leq 1}}\left\|L^{m} Z^{\alpha} u_{k-1}^{\prime}(s, \cdot)\right\|_{L^{2}\left(\mathbb{R}^{3}\right)} d s \\
\leq C \varepsilon^{2} \int_{0}^{T_{\varepsilon}} d s /(1+s) \leq C \cdot \kappa \cdot \varepsilon,
\end{array}
$$

where $\kappa$ is the constant appearing in (1.8). For the first term on the right-hand side in (3.18) we apply Lemma 3.4. If we fix $s$ and $R$, we note that, for $R / 2<|x|<2 R$,

$$
\sum_{\substack{|\alpha|+m \leq 5 \\ m \leq 1}}\left|L^{m} Z^{\alpha} u_{k-1}^{\prime}(s, x)\right| \leq C(1+R)^{-1} \sum_{\substack{|\alpha|+m \leq 7 \\ m \leq 1}}\left\|L^{m} Z^{\alpha} u_{k-1}^{\prime}(s, \cdot)\right\|_{L^{2}(R / 4<|x|<4 R)} .
$$

We can similarly bound this factor on the set $|x| \leq 1$. Therefore, for each fixed $s$ we have for a given $R=2^{j}, j \geq 0$,

$$
\begin{gathered}
\sum_{\substack{|\alpha|+m \leq 9 \\
m \leq 1}} \sum_{\substack{|\beta|+n \leq 5 \\
n \leq 1}}\left\|\left(L^{m} Z^{\alpha} u_{k-1}^{\prime}(s, \cdot)\right)\left(L^{n} Z^{\beta} u_{k-1}^{\prime}(s, \cdot)\right)\right\|_{L^{2}(R<|x|<2 R)} \\
\leq C 2^{-j} \sum_{\substack{|\alpha|+m \leq 9 \\
m \leq 1}}\left\|L^{m} Z^{\alpha} u_{k-1}^{\prime}(s, \cdot)\right\|_{L^{2}(R<|x|<2 R)} \\
\quad \times \sum_{\substack{|\alpha|+m \leq 5 \\
m \leq 1}}\left\|L^{m} Z^{\alpha} u_{k-1}^{\prime}(s, \cdot)\right\|_{L^{\infty}(R<|x|<2 R)} \\
\leq C \sum_{\substack{|\alpha|+m \leq 9 \\
m \leq 1}}\left\|\langle x\rangle^{-1 / 2} L^{m} Z^{\alpha} u_{k-1}^{\prime}(s, \cdot)\right\|_{L^{2}(R<|x|<2 R)},
\end{gathered}
$$


with a similar bound on the set $|x| \leq 1$, where we applied the Sobolev Lemma. Summing over $R=2^{j}$ and using the induction hypothesis, we conclude that

$$
\begin{gathered}
\int_{0}^{T_{\varepsilon}} \sum_{\substack{|\alpha|+m \leq 9 \\
m \leq 1}} \sum_{\substack{|\beta|+n \leq 5 \\
n \leq 1}}\left\|\left(L^{m} Z^{\alpha} u_{k-1}^{\prime}(s, \cdot)\right)\left(L^{n} Z^{\beta} u_{k-1}^{\prime}(s, \cdot)\right)\right\|_{L^{2}\left(\mathbb{R}^{3}\right)} d s \\
\leq C \int_{0}^{T_{\varepsilon}} \sum_{\substack{|\alpha|+m \leq 9 \\
m \leq 1}}\left\|\langle x\rangle^{-1 / 2} L^{m} Z^{\alpha} u_{k-1}^{\prime}(s, \cdot)\right\|_{L^{2}\left(\mathbb{R}^{3}\right)}^{2} \\
\leq C \ln \left(2+T_{\varepsilon}\right)\left(\sum_{\substack{|\alpha|+m \leq 9 \\
m \leq 1}}\left(\ln \left(2+T_{\varepsilon}\right)\right)^{-\frac{1}{2}}\left\|\langle x\rangle^{-1 / 2} L^{m} Z^{\alpha} u_{k-1}^{\prime}\right\|_{L^{2}\left(\left[0, T_{\varepsilon}\right) \times \mathbb{R}^{3}\right)}\right)^{2} \\
\leq C \cdot \kappa \cdot \varepsilon .
\end{gathered}
$$

We thus have shown that

$$
\int_{0}^{T_{\varepsilon}} \sum_{|\alpha|+m \leq 10, m \leq 1}\left\|L^{m} Z^{\alpha} B\left(u_{k-1}^{\prime}\right)(s, \cdot)\right\|_{L^{2}\left(\mathbb{R}^{3}\right)} d s \leq C \cdot \kappa \cdot \varepsilon .
$$

The second integral on the right side of (3.17) has a quasilinear contribution which is bounded by

$$
\begin{aligned}
& \int_{0}^{T_{\varepsilon}} \sum_{\substack{|\alpha|+m \leq 9 \\
m \leq 1}}\left\|u_{k-1}^{\prime}\left(L^{m} Z^{\alpha} u_{k}^{\prime \prime}(s, \cdot)\right)\right\|_{L^{2}\left(\mathbb{R}^{3}\right)} d s \\
& +\int_{0}^{T_{\varepsilon}} \sum_{\substack{|\alpha|+m \leq 5 \\
m \leq 1}} \sum_{\substack{|\beta|+n \leq 8 \\
n \leq 1}}\left\|\left(L^{m} Z^{\alpha} u_{k-1}^{\prime}(s, \cdot)\right)\left(L^{n} Z^{\beta} u_{k}^{\prime \prime}(s, \cdot)\right)\right\|_{L^{2}\left(\mathbb{R}^{3}\right)} d s \\
& +\int_{0}^{T_{\varepsilon}} \sum_{|\alpha|+m \leq 5} \sum_{\substack{|\beta|+n \leq 8 \\
n \leq 1}}\left\|\left(L^{m} Z^{\alpha} u_{k}^{\prime \prime}(s, \cdot)\right)\left(L^{n} Z^{\beta} u_{k-1}^{\prime}(s, \cdot)\right)\right\|_{L^{2}\left(\mathbb{R}^{3}\right)} d s \\
& +\int_{0}^{T_{\varepsilon}} \sum_{\substack{|\alpha|+m \leq 9 \\
m \leq 1}}\left\|u_{k}^{\prime \prime}(s, \cdot)\left(L^{m} Z^{\alpha} u_{k-1}^{\prime}(s, \cdot)\right)\right\|_{L^{2}\left(\mathbb{R}^{3}\right)} d s .
\end{aligned}
$$

We bound the integrand in the first integral of (3.23) by taking the first factor in $L^{\infty}$, the second factor in $L^{2}$, and arguing as in (3.19) above to bound this term by

$$
C \cdot \varepsilon \cdot M_{k}\left(T_{\varepsilon}\right) \int_{0}^{T_{\varepsilon}} \frac{1}{1+s} d s \leq C \cdot \kappa \cdot M_{k}\left(T_{\varepsilon}\right) .
$$

We estimate the second and third integrals in (3.23) as before, using the generalized Sobolev bound of Lemma 3.4 on the first factor. The fourth integral in (3.23) is bounded by taking the $u_{k}^{\prime \prime}$ factor in $L^{\infty}$, and arguing as before using the induction hypothesis. Both of these estimates yield bounds of $C \cdot \kappa \cdot M_{k}\left(T_{\varepsilon}\right)$.

The semilinear contribution from the second integral on the right of (3.17) is handled exactly as we bounded the first integral on the right of (3.17). 
To estimate the third integral in (3.17), which involves commutators, we begin by noting that

$$
\begin{aligned}
& \sum_{\substack{|\alpha|+m \leq 10 \\
m \leq 1}}\left|\left[L^{m} Z^{\alpha}, \gamma^{I J, i j}\left(u_{k-1}^{\prime}\right) \partial_{i} \partial_{j}\right] u_{k}\right| \\
& \leq C \sum_{\substack{|\alpha|+m \leq 9 \\
m \leq 1}}\left|L^{m} Z^{\alpha} u_{k-1}^{\prime}\right| \sum_{\substack{|\alpha|+m \leq 5 \\
m \leq 1}}\left|L^{m} Z^{\alpha} u_{k}^{\prime}\right| \\
& \quad+C \sum_{\substack{|\alpha|+m \leq 10 \\
m \leq 1}}\left|L^{m} Z^{\alpha} u_{k-1}^{\prime}\right| \cdot\left|u_{k}^{\prime \prime}\right| \\
& \quad+C \sum_{\substack{|\alpha|+m \leq 5 \\
m \leq 1}}\left|L^{m} Z^{\alpha} u_{k-1}^{\prime}\right| \sum_{\substack{|\alpha|+m \leq 9 \\
m \leq 1}}\left|L^{m} Z^{\alpha} u_{k}^{\prime}\right| \\
& \quad+C \sum_{|\alpha| \leq 1}\left|Z^{\alpha} u_{k-1}^{\prime}\right| \sum_{|\alpha|+m \leq 10}\left|L^{m} Z^{\alpha} u_{k}^{\prime}\right| \\
& \quad+C\left|L u_{k-1}^{\prime}\right| \sum_{|\alpha| \leq 9}\left|Z^{\alpha} u_{k}^{\prime \prime}\right| .
\end{aligned}
$$

The contribution of the first four terms to the third integral in (3.17) can be controlled as in the preceding arguments: when one factor appears with two or fewer $Z$ type derivatives, we take this factor out in $L^{\infty}$ as in (3.19) above; for the remaining terms we argue as in (3.20)-(3.22). The last term above requires a different argument since the factor we would like to take in $L^{\infty}$ now involves the scaling vector field $L$, which is not controlled by the term $I I I_{k-1}\left(T_{\varepsilon}\right)$ (see (3.15)).

To estimate this last term, let $c_{0}=\min _{I}\left\{c_{I}\right\}$. Then, on the region $|x|>c_{0} s / 4$, we can apply Lemma 3.4 to obtain

$$
\left|L u_{k-1}^{\prime}(s, x)\right| \leq C(1+s)^{-1} \sum_{|\alpha| \leq 2, m \leq 1}\left\|L^{m} Z^{\alpha} u_{k-1}^{\prime}(s, \cdot)\right\|_{L^{2}\left(\mathbb{R}^{3}\right)},
$$

and we conclude, as in (3.19),

$$
\int_{0}^{T_{\varepsilon}} \sum_{|\alpha| \leq 9}\left\|L u_{k-1}^{\prime}(s, \cdot) Z^{\alpha} u_{k}^{\prime \prime}(s, \cdot)\right\|_{L^{2}\left(|x|>c_{0} s / 4\right)} d s \leq C \cdot \kappa \cdot M_{k}\left(T_{\varepsilon}\right)
$$

It remains to estimate the integrand here on the region $|x| \leq c_{0} s / 4$. To do this, we bound the factor $L u_{k-1}^{\prime}$ in $L^{\infty}$ using Lemma 3.4 then apply Lemma 3.5 to $Z^{\alpha} u_{k}^{\prime \prime}$. 
We obtain, for $1 \leq R \leq c_{0} s / 4$,

$$
\begin{aligned}
& \sum_{|\alpha| \leq 9}\left\|L u_{k-1}^{\prime} Z^{\alpha} u_{k}^{\prime \prime}(s, \cdot)\right\|_{L^{2}(R / 2<|x|<R)} \\
& \leq C R^{-1} \sum_{|\alpha| \leq 2, m \leq 1}\left\|L^{m} Z^{\alpha} u_{k-1}^{\prime}(s, \cdot)\right\|_{L^{2}(R / 4<|x|<2 R)} \\
& \quad \times\left[\begin{array}{c}
(1+s)^{-1} \sum_{\substack{|\alpha|+m \leq 10 \\
m \leq 1}}\left\|L^{m} Z^{\alpha} u_{k}^{\prime}(s, \cdot)\right\|_{L^{2}(R / 4<|x|<2 R)} \\
+\sum_{|\alpha| \leq 9}\left\|Z^{\alpha} \square_{c} u_{k}(s, \cdot)\right\|_{L^{2}(R / 4<|x|<2 R)} \\
\left.+R^{-1} \sum_{|\alpha| \leq 9}\left(\left\|Z^{\alpha} u_{k}^{\prime}(s, \cdot)\right\|_{L^{2}(R / 4<|x|<2 R)}+\left\|Z^{\alpha} u_{k}(s, \cdot)\right\|_{L^{6}(R / 4<|x|<2 R)}\right)\right] .
\end{array}\right.
\end{aligned}
$$

We can control the norms over $|x|<1$ similarly. After squaring this estimate and summing over dyadic values of $R$, using extra factors of $R^{-1 / 2}$ to make the sums converge, we conclude that

$$
\begin{aligned}
\sum_{|\alpha| \leq 9} \int_{0}^{T_{\varepsilon}}\left\|L u_{k-1}^{\prime} Z^{\alpha} u_{k}^{\prime \prime}(s, \cdot)\right\|_{L^{2}\left(|x|<c_{0} s / 4\right)} d s \\
\leq C \int_{0}^{T_{\varepsilon}} \sum_{|\alpha| \leq 2, m \leq 1}\left\|L^{m} Z^{\alpha} u_{k-1}^{\prime}(s, \cdot)\right\|_{L^{2}\left(\mathbb{R}^{3}\right)}(1+s)^{-1} \\
\quad \times \sum_{|\alpha|+m \leq 10}\left\|L^{m} Z^{\alpha} u_{k}^{\prime}(s, \cdot)\right\|_{L^{2}\left(\mathbb{R}^{3}\right)} d s \\
\quad+\int_{0}^{T_{\varepsilon}} \sum_{|\alpha| \leq 2, m \leq 1}\left\|L^{m} Z^{\alpha} u_{k-1}^{\prime}(s, \cdot)\right\|_{L^{2}\left(\mathbb{R}^{3}\right)} \times \sum_{|\alpha| \leq 9}\left\|Z^{\alpha} \square_{c} u_{k}(s, \cdot)\right\|_{L^{2}\left(\mathbb{R}^{3}\right)} d s \\
\quad+\int_{0}^{T_{\varepsilon}} \sum_{|\alpha| \leq 2, m \leq 1}\left\|\langle x\rangle^{-1 / 2} L^{m} Z^{\alpha} u_{k-1}^{\prime}(s, \cdot)\right\|_{L^{2}\left(\mathbb{R}^{3}\right)} \\
\quad \times \sum_{|\alpha| \leq 9}\left(\left\|\langle x\rangle^{-1 / 2} Z^{\alpha} u_{k}^{\prime}(s, \cdot)\right\|_{L^{2}\left(\mathbb{R}^{3}\right)}+\left\|\langle x\rangle^{-1} Z^{\alpha} u_{k}(s, \cdot)\right\|_{L^{6}\left(\mathbb{R}^{3}\right)}\right) d s .
\end{aligned}
$$

The argument used in bounding the second integral in (3.17) yields

$$
\int_{0}^{T_{\varepsilon}} \sum_{|\alpha| \leq 9}\left\|Z^{\alpha} \square_{c} u_{k}(s, \cdot)\right\|_{L^{2}\left(\mathbb{R}^{3}\right)} d s \leq C \cdot \kappa \cdot\left(\varepsilon+M_{k}\left(T_{\varepsilon}\right)\right) .
$$


Plugging this into (3.24), and applying Cauchy-Schwarz to the $s$ integral of the last term on the right of (3.24), we conclude

$$
\begin{aligned}
\sum_{|\alpha| \leq 9} \int_{0}^{T_{\varepsilon}} \| L & u_{k-1}^{\prime} Z^{\alpha} u_{k}^{\prime \prime}(s, \cdot) \|_{L^{2}\left(|x|<c_{0} s / 4\right)} d s \\
& \leq \ln \left(2+T_{\varepsilon}\right) \cdot M_{k-1}\left(T_{\varepsilon}\right) \cdot M_{k}\left(T_{\varepsilon}\right)+M_{k-1}\left(T_{\varepsilon}\right) \cdot C \cdot \kappa \cdot\left(\varepsilon+M_{k}\left(T_{\varepsilon}\right)\right) \\
& \leq C \cdot \kappa \cdot\left(\varepsilon+M_{k}\left(T_{\varepsilon}\right)\right) .
\end{aligned}
$$

We have shown that

$$
I_{k}+I I_{k} \leq C_{0} \varepsilon+C \cdot \kappa \cdot\left(\varepsilon+M_{k}\left(T_{\varepsilon}\right)\right) .
$$

The final step is to show that $I I I_{k}\left(T_{\varepsilon}\right)$ can be controlled in this way,

$$
\sup _{0 \leq t \leq T_{\varepsilon}}(1+t) \sum_{|\alpha| \leq 1}\left|Z^{\alpha} u_{k}^{\prime}(t, x)\right| \leq C_{0} \varepsilon+C \cdot \kappa \cdot\left(\varepsilon+M_{k}\left(T_{\varepsilon}\right)\right)
$$

Together, (3.25) and (3.26) yield

$$
M_{k}\left(T_{\varepsilon}\right) \leq 3 C_{0} \varepsilon
$$

by choosing the constant $\kappa$ sufficiently small.

It suffices then to show (3.26). We first note that the left-hand side of (3.26) is bounded by

$$
C_{0} \varepsilon+\int_{0}^{t} \int_{\mathbb{R}^{3}} \sum_{|\alpha|+m \leq 5, m \leq 1}\left|L^{m} Z^{\alpha} \square_{c} u_{k}(s, y)\right| \frac{d y d s}{|y|} .
$$

This follows by Proposition [2.1, together with the fact that the Cauchy data of $Z^{\alpha} u_{k}^{\prime}$ at $t=0$ is of size $\varepsilon$ in the appropriate norm, and hence the homogeneous solution with the same Cauchy data satisfies the desired bounds (3.26), by the Klainerman-Sobolev inequalities [12.

We begin by handling the integral over $|y|>1$. We note that

$$
\begin{aligned}
& \sum_{|\alpha|+m \leq 5, m \leq 1}\left|L^{m} Z^{\alpha} \square_{c} u_{k}(s, y)\right| \\
& \leq C \sum_{\substack{|\alpha|+m \leq 7 \\
m \leq 1}}\left|L^{m} Z^{\alpha} u_{k-1}^{\prime}(s, y)\right| \sum_{\substack{|\alpha|+m \leq 7 \\
m \leq 1}}\left(\left|L^{m} Z^{\alpha} u_{k-1}^{\prime}(s, y)\right|+\left|L^{m} Z^{\alpha} u_{k}^{\prime}(s, y)\right|\right) \text {, }
\end{aligned}
$$


and conclude by the Schwarz inequality and the induction hypothesis that

$$
\begin{aligned}
& \int_{0}^{T_{\varepsilon}} \int_{|y|>1} \sum_{|\alpha|+m \leq 5, m \leq 1}\left|L^{m} Z^{\alpha} \square_{c} u_{k}(s, y)\right| \frac{d y d s}{|y|} \\
& \leq C\left(\sum_{\substack{|\alpha|+m \leq 7 \\
m \leq 1}}\left\|\langle y\rangle^{-\frac{1}{2}} L^{m} Z^{\alpha} u_{k-1}\right\|_{L^{2}\left(\left[0, T_{\varepsilon}\right] \times \mathbb{R}^{3}\right)}\right)^{2} \\
& +C\left(\sum_{\substack{|\alpha|+m \leq 7 \\
m \leq 1}}\left\|\langle y\rangle^{-\frac{1}{2}} L^{m} Z^{\alpha} u_{k-1}\right\|_{L^{2}\left(\left[0, T_{\varepsilon}\right] \times \mathbb{R}^{3}\right)}\right) \\
& \times\left(\sum_{\substack{|\alpha|+m \leq 7 \\
m \leq 1}}\left\|\langle y\rangle^{-\frac{1}{2}} L^{m} Z^{\alpha} u_{k}\right\|_{L^{2}\left(\left[0, T_{\varepsilon}\right] \times \mathbb{R}^{3}\right)}\right) \\
& \leq C \cdot \ln \left(2+T_{\varepsilon}\right) \cdot\left(M_{k-1}^{2}\left(T_{\varepsilon}\right)+M_{k-1}\left(T_{\varepsilon}\right) \cdot M_{k}\left(T_{\varepsilon}\right)\right) \\
& \leq C \cdot \kappa \cdot\left(\varepsilon+M_{k}\left(T_{\varepsilon}\right)\right),
\end{aligned}
$$

as desired.

To handle the integral over $|y|<1$, we apply the Sobolev inequality and (3.28) to obtain

$$
\begin{aligned}
& \sum_{|\alpha|+m \leq 5, m \leq 1} \sup _{|y|<1}\left|L^{m} Z^{\alpha} \square_{c} u_{k}(s, y)\right| \leq C \sum_{|\alpha|+m \leq 9, m \leq 1}\left\|L^{m} Z^{\alpha} u_{k-1}^{\prime}(s, \cdot)\right\|_{L^{2}(|y|<2)} \\
& \quad \times \sum_{|\alpha|+m \leq 9, m \leq 1}\left(|| L^{m} Z^{\alpha} u_{k-1}^{\prime}(s, \cdot)||_{L^{2}(|y|<2)}+\| L^{m} Z^{\alpha} u_{k}^{\prime}(s, \cdot)||_{L^{2}(|y|<2)}\right) .
\end{aligned}
$$

Since $\frac{1}{|y|} \in L^{1}\left(\mathbb{R}^{3}\right)$,

$$
\begin{aligned}
& \int_{0}^{T_{\varepsilon}} \int_{|y| \leq 1} \sum_{|\alpha| \leq 4, m \leq 1}\left|L^{m} Z^{\alpha} \square_{c} u_{k}(s, y)\right| \frac{d y d s}{|y|} \\
& \leq C\left(\sum_{\substack{|\alpha|+m \leq 9 \\
m \leq 1}}\left\|L^{m} Z^{\alpha} u_{k-1}^{\prime}\right\|_{L^{2}\left(\left[0, T_{\varepsilon}\right] \times\{|y| \leq 2\}\right)}\right)^{2} \\
& +C\left(\sum_{\substack{|\alpha|+m \leq 9 \\
m \leq 1}}\left\|L^{m} Z^{\alpha} u_{k-1}^{\prime}\right\|_{L^{2}\left(\left[0, T_{\varepsilon}\right] \times\{|y| \leq 2\}\right)}\right) \\
& \times\left(\sum_{|\alpha|+m \leq 9}^{m \leq 1}|| L^{m} Z^{\alpha} u_{k}^{\prime} \|_{L^{2}\left(\left[0, T_{\varepsilon}\right] \times\{|y| \leq 2\}\right)}\right) \\
& \leq C \cdot \kappa \cdot\left(\varepsilon+M_{k}\left(T_{\varepsilon}\right)\right)
\end{aligned}
$$

as above. We have therefore established (3.26).

Similar arguments show that

$$
\sup _{0 \leq t \leq T_{\varepsilon}}\left\|u_{k}^{\prime}(t, \cdot)-u_{k-1}^{\prime}(t, \cdot)\right\|_{L^{2}\left(\mathbb{R}^{3}\right)} \rightarrow 0, k \rightarrow \infty .
$$


We conclude that $u_{k}$ converges to a solution of (1.10) that verifies (3.13) with $C=3 C_{0}$. This completes the proof of Theorem 1.2.

Later we will need the following observation. If we replace the smallness condition (3.12) by

$$
\sum_{|\alpha| \leq N}\left\|\langle x\rangle^{|\alpha|} \partial_{x}^{\alpha} f^{\prime}\right\|_{L^{2}\left(\mathbb{R}^{3}\right)}+\sum_{|\alpha| \leq N-1}\left\|\langle x\rangle^{|\alpha|} \partial_{x}^{\alpha} g\right\|_{L^{2}\left(\mathbb{R}^{3}\right)} \leq \varepsilon,
$$

for $N \geq 10$, then the same argument as above gives that for $\varepsilon>0$ small, one obtains a solution on $\left[0, T_{\varepsilon}\right] \times \mathbb{R}^{3}$ verifying

$$
\begin{aligned}
\sup _{0 \leq t \leq T_{\varepsilon}} & \sum_{|\alpha|+m \leq N, m \leq 1}\left\|L^{m} Z^{\alpha} u^{\prime}(t, \cdot)\right\|_{L^{2}\left(\mathbb{R}^{3}\right)} \\
& +\left(\ln \left(2+T_{\varepsilon}\right)\right)^{-1 / 2} \sum_{|\alpha|+m \leq N-1, m \leq 1}\left\|L^{m} Z^{\alpha} u^{\prime}\right\|_{L^{2}\left(\left[0, T_{\varepsilon}\right] \times \mathbb{R}^{3}\right)} \leq C \varepsilon
\end{aligned}
$$

for this value of $N$.

\section{Pointwise estimates outside of Star-Shaped obstacles}

In this section we shall consider Dirichlet-wave equations outside of smooth, compact, star-shaped obstacles $\mathcal{K} \subset \mathbb{R}^{3}$. Our main goal is to show that the solution of the inhomogeneous equation

$$
\left\{\begin{array}{l}
\square u(t, x)=F(t, x), \quad(t, x) \in \mathbb{R}_{+} \times \mathbb{R}^{3} \backslash \mathcal{K}, \\
u(t, x)=0, \quad x \in \partial \mathcal{K}, \\
u(t, x)=0, \quad t \leq 0
\end{array}\right.
$$

satisfies slightly weaker pointwise estimates than those in Proposition 2.1 As before, $\square=\partial_{t}^{2}-\Delta$ denotes the unit-speed scalar D'Alembertian, and any of the following estimates for $\square$ extend to estimates for $\square_{c}$ after applying straightforward scaling arguments.

The pointwise estimate that we can prove is the following.

Theorem 4.1. Suppose that $\mathcal{K} \subset \mathbb{R}^{3}$ is a star-shaped obstacle as in (1.1). Then each $C^{\infty}$ solution $u$ of (4.1) satisfies, for each $\alpha$,

$$
\begin{aligned}
t\left|Z^{\alpha} u(t, x)\right| \leq C \int_{0}^{t} \int_{\mathbb{R}^{3} \backslash \mathcal{K}} & \sum_{\substack{|\beta|+j \leq|\alpha|+6 \\
j \leq 1}}\left|L^{j} Z^{\beta} F(s, y)\right| \frac{d y d s}{|y|} \\
& +C \int_{0}^{t} \sum_{\substack{|\beta|+j \leq|\alpha|+3 \\
j \leq 1}}\left\|L^{j} \partial_{s, y}^{\beta} F(s, \cdot)\right\|_{L^{2}\left(\mathbb{R}^{3} \backslash \mathcal{K}\right)} d s .
\end{aligned}
$$

As a first step, we shall see that for any obstacle, we can reduce things to proving decay estimates for $Z^{\alpha} u(t, x)$ when $x$ belongs to a fixed neighborhood of the obstacle. Here and in what follows, we shall assume, without loss of generality, that

$$
\mathcal{K} \subset\left\{x \in \mathbb{R}^{3}:|x|<1\right\}
$$


Lemma 4.2. Suppose that $u$ is as in Theorem 4.1 and that $\mathcal{K}$ satisfies (4.3). Then

$$
\begin{aligned}
(1+t)\left|Z^{\alpha} u(t, x)\right| \leq C & \int_{0}^{t} \int_{\mathbb{R}^{3} \backslash \mathcal{K}} \sum_{|\gamma|+j \leq 3, j \leq 1}\left|L^{j} Z^{\alpha+\gamma} F(s, y)\right| \frac{d y d s}{|y|} \\
& +C \sup _{|y|<2,0 \leq s \leq t}(1+s)\left(\left|Z^{\alpha} u^{\prime}(s, y)\right|+\left|Z^{\alpha} u(s, y)\right|\right) .
\end{aligned}
$$

Proof. The inequality is obvious for $|x|<2$. So we show that there is a uniform constant $C$ such that

$$
\begin{aligned}
(1+t) \sup _{|x| \geq 2}\left|Z^{\alpha} u(t, x)\right| \leq & C \int_{0}^{t} \int_{\mathbb{R}^{3} \backslash \mathcal{K}} \sum_{|\gamma|+j \leq 3, j \leq 1}\left|L^{j} Z^{\alpha+\gamma} F(s, y)\right| \frac{d y d s}{|y|} \\
& +C \sup _{|y|<2,0 \leq s \leq t}(1+s)\left(\left|Z^{\alpha} u^{\prime}(s, y)\right|+\left|Z^{\alpha} u(s, y)\right|\right) .
\end{aligned}
$$

For this, we fix $\rho \in C^{\infty}(\mathbb{R})$ satisfying $\rho(r)=1, r \geq 2$ and $\rho(r)=0, r \leq 1$. Then

$$
w(t, x)=\rho(|x|) Z^{\alpha} u(t, x)
$$

solves the boundaryless wave equation

$$
\square w(t, x)=\rho Z^{\alpha} F(t, x)-2 \rho^{\prime}(|x|) \frac{x}{|x|} \cdot \nabla_{x} Z^{\alpha} u(t, x)-\left(\Delta \rho(|x|) Z^{\alpha} u(t, x),\right.
$$

with zero initial data. We split $w=w_{0}+w_{1}$, where $\square w_{1}=\rho Z^{\alpha} F$. If we apply Proposition 2.1 we conclude that $(1+t)\left|w_{1}(t, x)\right|$ is dominated by the first term in the right side of (4.5), and so it suffices to show that $(1+t)\left|w_{0}(t, x)\right|$ is dominated by the last term in (4.5). Write

$$
G(t, x)=-2 \rho^{\prime}(|x|) \frac{x}{|x|} \cdot \nabla_{x} Z^{\alpha} u(t, x)-(\Delta \rho(|x|)) Z^{\alpha} u(t, x) .
$$

By Lemma 2.2

$$
\left|w_{0}(t, x)\right| \leq C \frac{1}{|x|} \int_{0}^{t} \int_{|| x|-(t-s)||\theta|=1}^{|x|+(t-s)} \sup _{|\theta|=1}|G(s, r \theta)| r d r d s .
$$

However, $G(t, x)=0$ for $|x| \leq 1$ and $|x| \geq 2$. Hence the $s$ integrand in (4.6) is nonzero only when

$$
-2 \leq|x|-(t-s) \leq 2
$$

that is,

$$
(t-|x|)-2 \leq s \leq(t-|x|)+2 .
$$

We conclude that

$$
\begin{aligned}
\left|w_{0}(t, x)\right| \leq & C \frac{1}{|x|} \frac{1}{1+|t-| x||} \\
& \times \sup _{\substack{(t-|x|-2) \leq s \leq(t-|x|+2) \\
|y| \leq 2}}(1+s)\left(\left|Z^{\alpha} u^{\prime}(s, y)\right|+\left|Z^{\alpha} u(s, y)\right|\right) .
\end{aligned}
$$

This yields immediately the desired bounds for $\left|w_{0}(t, x)\right|$ and completes the proof of Lemma 4.2.

To establish decay estimates for $|x|<2$ we shall use the following local energy estimates, which follow from the exponential decay estimates of Lax, Morawetz, and Phillips (see [16], also [21] for local exponential decay outside more general obstacles). 
Lemma 4.3. Suppose that $u \in C^{\infty}$ satisfies (4.1), where $\mathcal{K} \subset \mathbb{R}^{3}$ is a star-shaped obstacle as in (4.3). Suppose also that $F(t, x)=0$ for $|x|>4$. Then there is a constant $c>0$ so that

$$
\left\|u^{\prime}(t, \cdot)\right\|_{L^{2}\left(\mathbb{R}^{3} \backslash \mathcal{K}:|x|<4\right)} \leq C \int_{0}^{t} e^{-c(t-s)}\|F(s, \cdot)\|_{L^{2}\left(\mathbb{R}^{3} \backslash \mathcal{K}\right)} d s .
$$

Consequently, under these assumptions, if $M=0,1,2, \ldots$ is fixed,

$$
\begin{aligned}
\sum_{\substack{|\alpha|+j \leq M \\
j \leq 1}}\left\|\left(t \partial_{t}\right)^{j} \partial_{t, x}^{\alpha} u^{\prime}(t, \cdot)\right\|_{L^{2}\left(\mathbb{R}^{3} \backslash \mathcal{K}:|x|<4\right)} & \\
\leq & C \sum_{\substack{|\alpha|+j \leq M-1 \\
j \leq 1}}\left\|\left(t \partial_{t}\right)^{j} \partial_{t, x}^{\alpha} F(t, \cdot)\right\|_{L^{2}\left(\mathbb{R}^{3} \backslash \mathcal{K}\right)} \\
& +C \int_{0}^{t} e^{-\frac{c}{2}(t-s)} \sum_{\substack{|\alpha|+j \leq M \\
j \leq 1}}\left\|\left(s \partial_{s}\right)^{j} \partial_{s, x}^{\alpha} F(s, \cdot)\right\|_{L^{2}\left(\mathbb{R}^{3} \backslash \mathcal{K}\right)} d s .
\end{aligned}
$$

Proof. The first estimate is an immediate consequence of the exponential decay estimates of Lax and Phillips. As for (4.8), using induction and elliptic regularity (see the proof of Theorem 5.2 below) one shows that for all $M=0,1,2, \ldots$,

$$
\begin{aligned}
\sum_{|\alpha| \leq M}\left\|\partial_{t, x}^{\alpha} u^{\prime}(t, \cdot)\right\|_{L^{2}\left(\mathbb{R}^{3} \backslash \mathcal{K}:|x|<4\right)} & \leq C \sum_{|\alpha| \leq M-1}\left\|\partial_{t, x}^{\alpha} F(t, \cdot)\right\|_{L^{2}\left(\mathbb{R}^{3}\right)} \\
& +C \int_{0}^{t} e^{-c(t-s)} \sum_{|\alpha| \leq M}\left\|\partial_{s, x}^{\alpha} F(s, \cdot)\right\|_{L^{2}\left(\mathbb{R}^{3}\right)} d s .
\end{aligned}
$$

It remains to bound

$$
\sum_{|\alpha| \leq M-1}\left\|\left(t \partial_{t}\right) \partial_{t, x}^{\alpha} u^{\prime}(t, \cdot)\right\|_{L^{2}\left(\mathbb{R}^{3} \backslash \mathcal{K}:|x|<4\right)} .
$$

Clearly $\partial_{t} u$ satisfies (4.1) with forcing term $\partial_{t} F$. Apply (4.9) to this equation for $\partial_{t} u$, summing on the left over $|\alpha| \leq M-1$, and multiply both sides of the resulting inequality by $t$ to bound (4.10) as in (4.8).

For later use, notice that since $L=t \partial_{t}+r \partial_{r}$, inequality (4.8) implies that if $F(s, y)=0,|y|>4$, then

$$
\begin{gathered}
\sum_{|\alpha|+j \leq M, j \leq 1}\left\|\left(t \partial_{t}\right)^{j} \partial_{t, x}^{\alpha} u^{\prime}(t, \cdot)\right\|_{L^{2}\left(\mathbb{R}^{3} \backslash \mathcal{K}:|x|<4\right)} \leq C \sum_{\substack{|\alpha|+j \leq M-1 \\
j \leq 1}}\left\|L^{j} \partial_{t, x}^{\alpha} F(t, \cdot)\right\|_{L^{2}\left(\mathbb{R}^{3}\right)} \\
+C \int_{0}^{t} e^{-\frac{c}{2}(t-s)} \sum_{\substack{|\alpha|+j \leq M \\
j \leq 1}}\left\|L^{j} \partial_{s, x}^{\alpha} F(s, \cdot)\right\|_{L^{2}\left(\mathbb{R}^{3}\right)} d s .
\end{gathered}
$$

End of proof of Theorem 4.1. Since the coefficients of $Z$ are bounded when $|x|<2$, it suffices to show that if $|\beta| \leq|\alpha|+1$ (where $\alpha$ was fixed in the statement of the 
Theorem), then

$$
\begin{aligned}
t \sup _{|x|<2}\left|\partial_{t, x}^{\beta} u(t, x)\right| \leq C \int_{0}^{t} & \sum_{\substack{|\gamma|+j \leq|\alpha|+3 \\
j \leq 1}}\left\|L^{j} \partial_{s, x}^{\gamma} F(s, \cdot)\right\|_{L^{2}\left(\mathbb{R}^{3} \backslash \mathcal{K}\right)} d s \\
& +C \sum_{\substack{|\gamma|+j \leq|\alpha|+4 \\
j \leq 1,|\mu| \leq 2}} \int_{0}^{t} \int\left|L^{j} \Omega^{\mu} \partial_{s, x}^{\gamma} F(s, y)\right| \frac{d y d s}{|y|} .
\end{aligned}
$$

Using cutoffs for the forcing terms, we can split things into proving (4.12) for the following two cases:

- Case 1: $F(s, y)=0$ if $|y|>4$,

- Case 2: $F(s, y)=0$ if $|y|<3$.

For either case, we shall use the following immediate consequence of the fundamental theorem of calculus,

$$
\left|t \partial_{t, x}^{\beta} u(t, x)\right| \leq \int_{0}^{t} \sum_{j \leq 1}\left|\left(s \partial_{s}\right)^{j} \partial_{s, x}^{\beta} u(s, x)\right| d s .
$$

We apply the Sobolev Lemma to the right side, using the fact that $|\beta| \leq|\alpha|+1$, and that Dirichlet conditions allow us to control $u$ locally by $u^{\prime}$, to conclude that

$$
\begin{aligned}
t \sup _{|x|<2}\left|\partial_{t, x}^{\beta} u(t, x)\right| & \leq C \int_{0}^{t} \sum_{|\gamma| \leq|\alpha|+2, j \leq 1}\left\|\left(s \partial_{s}\right)^{j} \partial_{s, x}^{\gamma} u^{\prime}(s, \cdot)\right\|_{L^{2}\left(\mathbb{R}^{3} \backslash \mathcal{K}:|x|<4\right)} d s \\
& \leq C \int_{0}^{t} \sum_{|\gamma|+j \leq|\alpha|+3, j \leq 1}\left\|\left(s \partial_{s}\right)^{j} \partial_{s, x}^{\gamma} u^{\prime}(s, \cdot)\right\|_{L^{2}\left(\mathbb{R}^{3} \backslash \mathcal{K}:|x|<4\right)} d s .
\end{aligned}
$$

If we are in Case 1, we can apply (4.11) to get 4.12).

In Case 2, we need to write $u=u_{0}+u_{r}$ where $u_{0}$ solves the boundaryless wave equation $\square u_{0}=F$ with zero initial data. Fix $\eta \in C_{0}^{\infty}\left(\mathbb{R}^{3}\right)$ satisfying $\eta(x)=1$, $|x|<2$, and $\eta(x)=0,|x| \geq 3$. It follows that if we set $\tilde{u}=\eta u_{0}+u_{r}$ then, since $\eta F=0, \tilde{u}$ solves the Dirichlet-wave equation

$$
\square \tilde{u}=G=-2 \nabla_{x} \eta \cdot \nabla_{x} u_{0}-(\Delta \eta) u_{0}
$$

with zero initial data. The forcing term $G$ vanishes unless $2 \leq|x| \leq 4$. Hence by Case 1,

$$
\begin{aligned}
& t \sup _{|x|<2}\left|\partial_{t, x}^{\beta} u(t, x)\right|=t \sup _{|x|<2}\left|\partial_{t, x}^{\beta} \tilde{u}(t, x)\right| \\
& \quad \leq C \int_{0}^{t} \sum_{|\gamma|+j \leq|\alpha|+3, j \leq 1}\left\|L^{j} \partial_{s, x}^{\gamma} G(s, \cdot)\right\|_{L^{2}\left(\mathbb{R}^{3} \backslash \mathcal{K}\right)} d s \\
& \quad \leq C \int_{0}^{t} \sum_{|\gamma|+j \leq|\alpha|+4, j \leq 1}\left\|L^{j} \partial_{s, x}^{\gamma} u_{0}(s, \cdot)\right\|_{L^{2}(2 \leq|x| \leq 4)} d s \\
& \quad \leq C \int_{0}^{t} \sum_{|\gamma|+j \leq|\alpha|+4, j \leq 1}\left\|L^{j} \partial_{s, x}^{\gamma} u_{0}(s, \cdot)\right\|_{L^{\infty}(2 \leq|x| \leq 4)} d s .
\end{aligned}
$$


To finish the argument, we apply (2.5) to $w=L^{j} \partial_{s, x}^{\gamma} u_{0}$ with $j=0,1$. Doing so yields

$$
\begin{aligned}
\left\|L^{j} \partial_{s, x}^{\gamma} u_{0}(s, \cdot)\right\|_{L^{\infty}(2 \leq|x| \leq 4)} & \leq C \int_{0}^{s} \int_{|s-\tau-\rho| \leq 4} \sup _{|\theta|=1}\left|L^{j} \partial_{\tau, x}^{\gamma} F(\tau, \rho \theta)\right| \rho d \rho d \tau \\
& \leq C \sum_{|\mu| \leq 2} \int_{0}^{s} \int_{|s-\tau-\rho| \leq 4}\left|L^{j} \partial_{\tau, x}^{\gamma} \Omega^{\mu} F(\tau, \rho \theta)\right| \rho d \rho d \theta d \tau \\
& =C \sum_{|\mu| \leq 2} \int_{0}^{s} \int_{|s-\tau-| y|| \leq 4}\left|L^{j} \partial_{\tau, x}^{\gamma} \Omega^{\mu} F(\tau, y)\right| \frac{d y d \tau}{|y|}
\end{aligned}
$$

Note that the sets $\Lambda_{s}=\{(\tau, y): 0 \leq \tau \leq s,|s-\tau-| y|| \leq 4\}$ satisfy $\Lambda_{s} \cap \Lambda_{s^{\prime}}=\emptyset$ if $\left|s-s^{\prime}\right|>20$. Therefore, if in the preceding inequality we sum over $|\gamma|+j \leq|\alpha|+4$, $j \leq 1$ and then integrate over $s \in[0, t]$ we conclude that 4.12) must also hold for Case 2, which completes the proof.

\section{Fixed time $L^{2}$ estimates for EuClidean Vector fields OUTSIDE OBSTACLES}

In this section we shall work with wave equations that are small perturbations of the standard D'Alembertian $\square$ on $\mathbb{R}_{+} \times \mathbb{R}^{3} \backslash \mathcal{K}$. We let $\square_{\gamma}$ denote the second-order operator given by

$$
\left(\square_{\gamma} w\right)^{I}=\left(\partial_{t}^{2}-c_{I}^{2} \Delta\right) w^{I}+\sum_{J=1}^{N} \sum_{j, k=0}^{3} \gamma^{I J, j k}(t, x) \partial_{j} \partial_{k} w^{J}, \quad 1 \leq I \leq N,
$$

where the perturbation terms $\gamma^{I J, j k}$ satisfy the symmetry conditions (3.6). Given $T>0$ fixed, we shall assume that $\gamma$ is uniformly small,

$$
\sum_{I, J=1}^{N} \sum_{j, k=0}^{3}\left\|\gamma^{I J, j k}(t, x)\right\|_{L^{\infty}\left([0, T] \times \mathbb{R}^{3} \backslash \mathcal{K}\right)} \leq \delta,
$$

and we also assume that

$$
\sum_{I, J=1}^{N} \sum_{i, j, k=0}^{3}\left\|\partial_{i} \gamma^{I J, j k}(t, x)\right\|_{L_{t}^{1} L_{x}^{\infty}\left([0, T] \times \mathbb{R}^{3} \backslash \mathcal{K}\right)} \leq C_{0} .
$$

Under these assumptions we shall prove $L^{2}$ estimates for solutions of the inhomogeneous Dirichlet-wave equation

$$
\left\{\begin{array}{l}
\square_{\gamma} w=F \\
\left.w\right|_{\partial \mathcal{K}}=0, \\
w(t, x)=0, \quad t \leq 0 .
\end{array}\right.
$$

The first estimate is the standard energy estimate:

Theorem 5.1. Assume $w \in C^{2}$ satisfy (5.4), and $\gamma$ as above satisfies the symmetry conditions (3.6) as well as (5.3) and (5.2) for $\delta>0$ sufficiently small. Then

$$
\left\|w^{\prime}(t, \cdot)\right\|_{L^{2}\left(\mathbb{R}^{3} \backslash \mathcal{K}\right)} \leq C \int_{0}^{t}\|F(s, \cdot)\|_{L^{2}\left(\mathbb{R}^{3} \backslash \mathcal{K}\right)} d s, \quad 0 \leq t \leq T,
$$

for a uniform constant $C$ (depending on $C_{0}$ ). 
Although the result is standard, we shall present its proof since it serves as a model for the more technical variations which are to follow.

We first define the components of the energy-momentum vector. For $I=$ $1,2, \ldots, N$, we let

$$
\begin{aligned}
e_{0}^{I}=e_{0}^{I}(w)=\left(\partial_{0} w^{I}\right)^{2} & +\sum_{k=1}^{3} c_{I}^{2}\left(\partial_{k} w^{I}\right)^{2} \\
& +2 \sum_{J=1}^{N} \sum_{k=0}^{3} \gamma^{I J, 0 k} \partial_{0} w^{I} \partial_{k} w^{J}-\sum_{J=1}^{N} \sum_{j, k=0}^{3} \gamma^{I J, j k} \partial_{j} w^{I} \partial_{k} w^{J}
\end{aligned}
$$

and for $k=1,2,3$,

$$
e_{k}^{I}=e_{k}^{I}(w)=-2 c_{I}^{2} \partial_{0} w^{I} \partial_{k} w^{I}+2 \sum_{J=1}^{N} \sum_{j=0}^{3} \gamma^{I J, j k} \partial_{0} w^{I} \partial_{j} w^{J}
$$

Then

$$
\begin{aligned}
\partial_{0} e_{0}^{I}= & 2 \partial_{0} w^{I} \partial_{0}^{2} w^{I}+2 \sum_{k=1}^{3} c_{I}^{2} \partial_{k} w^{I} \partial_{0} \partial_{k} w^{I} \\
& +2 \partial_{0} w^{I} \sum_{J=1}^{N} \sum_{k=0}^{3} \gamma^{I J, 0 k} \partial_{0} \partial_{k} w^{J}+2 \sum_{J=1}^{N} \sum_{k=0}^{3} \gamma^{I J, 0 k} \partial_{0}^{2} w^{I} \partial_{k} w^{J} \\
& -\sum_{J=1}^{N} \sum_{j, k=0}^{3} \gamma^{I J, j k}\left[\partial_{0} \partial_{j} w^{I} \partial_{k} w^{J}+\partial_{j} w^{I} \partial_{0} \partial_{k} w^{J}\right]+R_{0}^{I}
\end{aligned}
$$

where

$$
R_{0}^{I}=2 \sum_{J=1}^{N} \sum_{k=0}^{3}\left(\partial_{0} \gamma^{I J, 0 k}\right) \partial_{0} w^{I} \partial_{k} w^{J}-\sum_{J=1}^{N} \sum_{j, k=0}^{3}\left(\partial_{0} \gamma^{I J, j k}\right) \partial_{j} w^{I} \partial_{k} w^{J}
$$

Also,

$$
\begin{aligned}
\sum_{k=1}^{3} \partial_{k} e_{k}^{I}= & -2 \partial_{0} w^{I} c_{I}^{2} \Delta w^{I}-2 \sum_{k=1}^{3} c_{I}^{2} \partial_{k} w^{I} \partial_{0} \partial_{k} w^{I} \\
& +2 \partial_{0} w^{I} \sum_{J=1}^{N} \sum_{j=0}^{3} \sum_{k=1}^{3} \gamma^{I J, j k} \partial_{j} \partial_{k} w^{J} \\
& +2 \sum_{J=1}^{N} \sum_{j=0}^{3} \sum_{k=1}^{3} \gamma^{I J, j k} \partial_{0} \partial_{k} w^{I} \partial_{j} w^{J}+\sum_{k=1}^{3} R_{k}^{I},
\end{aligned}
$$

where

$$
R_{k}^{I}=2 \sum_{J=1}^{N} \sum_{k=1}^{3}\left(\partial_{k} \gamma^{I J, j k}\right) \partial_{0} w^{I} \partial_{j} w^{J}
$$

Note that by the symmetry conditions (3.6), if we sum the second to last term and the third to last term in (5.8) over $I$, we get

$$
-2 \sum_{I, J=1}^{N} \sum_{j=0}^{3} \sum_{k=1}^{3} \gamma^{I J, j k} \partial_{0} \partial_{k} w^{I} \partial_{j} w^{J}
$$


which is -1 times the sum over $I$ of the second to last term of (5.9). From this, we conclude that if we set

$$
e_{j}=e_{j}(w)=\sum_{I=1}^{N} e_{j}^{I}, \quad j=0,1,2,3,
$$

and

$$
R=R\left(w^{\prime}, w^{\prime}\right)=\sum_{I=1}^{N} \sum_{k=0}^{3} R_{k}^{I},
$$

then

$$
\partial_{t} e_{0}+\sum_{k=1}^{3} \partial_{k} e_{k}=2\left\langle\partial_{t} w, \square_{\gamma} w\right\rangle+R\left(w^{\prime}, w^{\prime}\right),
$$

with $\langle\cdot, \cdot\rangle$ denoting the standard inner product in $\mathbb{R}^{N}$.

If we integrate this identity over $\mathbb{R}^{3} \backslash \mathcal{K}$ and apply the divergence theorem, we obtain

$$
\begin{aligned}
\partial_{t} \int_{\mathbb{R}^{3} \backslash \mathcal{K}} e_{0}(t, x) d x-\int_{\partial \mathcal{K}} & \sum_{j=1}^{3} e_{j} n_{j} d \sigma \\
& =2 \int_{\mathbb{R}^{3} \backslash \mathcal{K}}\left\langle\partial_{t} w, \square_{\gamma} w\right\rangle d x+\int_{\mathbb{R}^{3} \backslash \mathcal{K}} R\left(w^{\prime}, w^{\prime}\right) d x .
\end{aligned}
$$

Here, $\vec{n}$ is the outward normal to $\mathcal{K}$, and $d \sigma$ is surface measure on $\partial \mathcal{K}$.

Since we are assuming that $w$ solves (5.4), and hence $\partial_{t} w$ vanishes on $\partial \mathcal{K}$, the integrand in the last term in the left side of (5.10) vanishes identically. Therefore, we have

$$
\partial_{t} \int_{\mathbb{R}^{3} \backslash \mathcal{K}} e_{0}(t, x) d x=2 \int_{\mathbb{R}^{3} \backslash \mathcal{K}}\left\langle\partial_{t} w, F\right\rangle d x+\int_{\mathbb{R}^{3} \backslash \mathcal{K}} R\left(w^{\prime}, w^{\prime}\right) d x .
$$

Note that if $\delta$ in (5.2) is small, then

$$
\left(2 \max _{I}\left\{c_{I}^{2}, c_{I}^{-2}\right\}\right)^{-1}\left|w^{\prime}(t, x)\right|^{2} \leq e_{0}(t, x) \leq 2 \max _{I}\left\{c_{I}^{2}, c_{I}^{-2}\right\}\left|w^{\prime}(t, x)\right|^{2} .
$$

This yields

$$
\begin{aligned}
& \partial_{t}\left(\int_{\mathbb{R}^{3} \backslash \mathcal{K}} e_{0}(t, x) d x\right)^{1 / 2} \\
& \leq C\|F(t, \cdot)\|_{L^{2}\left(\mathbb{R}^{3} \backslash \mathcal{K}\right)}+C \sum_{I, J=1}^{N} \sum_{i, j, k=0}^{3}\left\|\partial_{i} \gamma^{I J, j k}(t, \cdot)\right\|_{\infty}\left(\int_{\mathbb{R}^{3} \backslash \mathcal{K}} e_{0}(t, x) d x\right)^{1 / 2} .
\end{aligned}
$$

The theorem now follows from (5.11), (5.3), and Gronwall's inequality.

We will also need the following estimates for $L^{2}$ norms of higher-order derivatives.

Theorem 5.2. Suppose that $\gamma^{I J, j k} \in C^{\infty}\left([0, T] \times \mathbb{R}^{3} \backslash \mathcal{K}\right)$ satisfy the symmetry conditions (3.6) as well as (5.2) and (5.3) where $0<\delta<1 / 2$ in (5.2) is small enough so that (5.5) holds. Then if $w$ solves (5.4) and if $N=0,1,2, \ldots$ there is a 
constant $C$, depending on $N, \delta, \mathcal{K}$, and $C_{0}$, so that for $0<t<T$,

$$
\begin{aligned}
\sum_{|\alpha| \leq N}\left\|\partial_{t, x}^{\alpha} w^{\prime}(t, \cdot)\right\|_{L^{2}\left(\mathbb{R}^{3} \backslash \mathcal{K}\right)} & \leq C \int_{0}^{t} \sum_{j \leq N}\left\|\square_{\gamma} \partial_{s}^{j} w(s, \cdot)\right\|_{L^{2}\left(\mathbb{R}^{3} \backslash \mathcal{K}\right)} d s \\
& +C \sum_{|\alpha| \leq N-1}\left\|\square_{c} \partial_{t, x}^{\alpha} w(t, \cdot)\right\|_{L^{2}\left(\mathbb{R}^{3} \backslash \mathcal{K}\right)},
\end{aligned}
$$

where $\square_{\gamma}$ and $\square_{c}$ are as in (5.1) and (1.3), respectively.

Proof of Theorem 5.2, We have already observed that (5.12) holds when $N=0$. So we show that if the estimate is valid if $N$ is replaced by $N-1$, then it must be valid for $N$.

We first observe that, since $\left.\partial_{t} w\right|_{\mathbb{R}_{+} \times \partial \mathcal{K}}=0, \sum_{|\alpha|<N-1}\left\|\partial_{t, x}^{\alpha}\left(\partial_{t} w\right)^{\prime}(t, \cdot)\right\|_{L^{2}\left(\mathbb{R}^{3}\right)}$ is dominated by the right side of (5.12). Hence it suffices to show that, for $N \geq 1$,

$$
\sum_{|\alpha|=N}\left\|\partial_{x}^{\alpha} \nabla_{x} w(t, \cdot)\right\|_{L^{2}\left(\mathbb{R}^{3}\right)}
$$

also has this property. But,

$$
\begin{aligned}
& \sum_{|\alpha|=N-1}\left\|\Delta \partial_{x}^{\alpha} w(t, \cdot)\right\|_{L^{2}\left(\mathbb{R}^{3}\right)} \\
& \quad \leq C \sum_{|\alpha|=N-1}\left\|\partial_{x}^{\alpha} \partial_{t}^{2} w(t, \cdot)\right\|_{L^{2}\left(\mathbb{R}^{3}\right)}+C \sum_{|\alpha|=N-1}\left\|\square_{c} \partial_{x}^{\alpha} w(t, \cdot)\right\|_{L^{2}\left(\mathbb{R}^{3}\right)},
\end{aligned}
$$

where $C$ depends only on the wave speeds, $c_{I}$. As we have observed, the first term in the right side of (5.13) is dominated by the right side of (5.12), and thus the left side of (5.13) is similarly bounded. By elliptic regularity, so is $\sum_{|\alpha|=N}\left\|\partial_{x}^{\alpha} \nabla_{x} w(t, \cdot)\right\|_{L^{2}\left(\mathbb{R}^{3}\right)}$, which completes the proof.

\section{Weighted $L_{t}^{2} L_{x}^{2}$ estimates for the D'Alembertian OUTSIDE STAR-SHAPED OBSTACLES}

We shall also require $L_{t}^{2} L_{x}^{2}$ estimates for the unperturbed inhomogeneous wave equation near the obstacle. As in section 4 , we consider the scalar Dirichlet-wave equation, where $\square=\partial_{t}^{2}-\Delta$,

$$
\left\{\begin{array}{l}
\square v=G, \\
\left.v\right|_{\partial \mathcal{K}}=0, \\
v(t, \cdot)=0, \quad t<0 .
\end{array}\right.
$$

Just as before, our estimates here extend to solutions of non-unit speed scalar wave equations after a straightforward scaling argument. One of the required estimates is the following.

Proposition 6.1. Let $v$ be as in (6.1). Assume also that $\mathcal{K}$ is star-shaped and contained in $\left\{x \in \mathbb{R}^{3}:|x|<1\right\}$. Then there is a uniform constant $C$ so that

$$
\left\|v^{\prime}\right\|_{L^{2}\left([0, t] \times \mathbb{R}^{3} \backslash \mathcal{K}:|x|<2\right)} \leq C \int_{0}^{t}\|\square v(s, \cdot)\|_{L^{2}\left(\mathbb{R}^{3} \backslash \mathcal{K}\right)} d s .
$$


Additionally, if $N=1,2,3, \ldots$ is fixed, there is a uniform constant $C$ so that

$$
\begin{aligned}
& \sum_{|\alpha| \leq N}\left\|\partial_{s, x}^{\alpha} v^{\prime}\right\|_{L^{2}\left([0, t] \times \mathbb{R}^{3} \backslash \mathcal{K}:|x|<2\right)} \\
& \leq C \int_{0}^{t} \sum_{m \leq N}\left\|\square \partial_{s}^{m} v(s, \cdot)\right\|_{L^{2}\left(\mathbb{R}^{3} \backslash \mathcal{K}\right)} d s+C \sum_{|\alpha| \leq N-1}\left\|\square \partial_{s, x}^{\alpha} v\right\|_{L^{2}\left([0, t] \times \mathbb{R}^{3} \backslash \mathcal{K}\right)} .
\end{aligned}
$$

Proof. The elliptic regularity argument used in the proof of Theorem 5.2 shows that (6.3) is a consequence of (6.2); so we shall prove only (6.2).

To prove (6.2), we consider first the case when $G(s, y)=0$ for $|y|>4$. In this case, (4.7) and the Schwarz inequality give us for $0<\tau<t$,

$$
\begin{aligned}
& \left\|v^{\prime}(\tau, \cdot)\right\|_{L^{2}\left(\mathbb{R}^{3} \backslash \mathcal{K}:|x|<2\right)}^{2} \\
& \quad \leq C\left(\int_{0}^{\tau} e^{-c(\tau-s)}\|G(s, \cdot)\|_{L^{2}\left(\mathbb{R}^{3} \backslash \mathcal{K}\right)} d s\right)\left(\int_{0}^{t}\|G(s, \cdot)\|_{L^{2}\left(\mathbb{R}^{3} \backslash \mathcal{K}\right)} d s\right) .
\end{aligned}
$$

This implies (6.3) after integrating $\tau$ from 0 to $t$. Note, in addition, that applying the Schwarz inequality to (4.7) in a slightly different way yields

$$
\begin{aligned}
\left\|v^{\prime}(\tau, \cdot)\right\|_{L^{2}\left([0, t] \times \mathbb{R}^{3} \backslash \mathcal{K}:|x|<2\right)}^{2} & \leq C \int_{0}^{t}\left(\int_{0}^{\tau} e^{-c(\tau-s)}\|G(s, \cdot)\|_{L^{2}\left(\mathbb{R}^{3} \backslash \mathcal{K}\right)} d s\right)^{2} d \tau \\
& \leq C \int_{0}^{t} \int_{0}^{\tau} e^{-\frac{c}{2}(\tau-s)}\|G(s, \cdot)\|_{L^{2}\left(\mathbb{R}^{3} \backslash \mathcal{K}\right)}^{2} d s d \tau
\end{aligned}
$$

again under the assumption that $G(s, y)=\square v(s, y)=0,|y|>4$. Therefore, we also have

$$
\left\|v^{\prime}\right\|_{L^{2}\left(\mathbb{R}^{3} \backslash \mathcal{K}:|x|<2\right)} \leq C\|G\|_{L^{2}\left([0, t] \times \mathbb{R}^{3} \backslash \mathcal{K}\right)}, \quad \text { if } G(s, y)=0,|y|>4 .
$$

To finish, we need to show that we also have (6.2) when we assume that $G(s, y)=$ $\square v(s, y)=0,|y|<3$. For this, as in the proof of Theorem 4.1 we fix $\eta \in C^{\infty}\left(\mathbb{R}^{3}\right)$ satisfying $\eta(x)=1,|x| \leq 2$, and $\eta(x)=0,|x| \geq 3$. Then if we write $v=v_{0}+v_{r}$, where $v_{0}$ solves the boundaryless wave equation $\square v_{0}=G$ with zero initial data, it follows that $\tilde{v}=\eta v_{0}+v_{r}$ solves the Dirichlet-wave equation

$$
\square \tilde{v}=\tilde{G}=-2 \nabla_{x} \eta \cdot \nabla_{x} v_{0}-(\Delta \eta) v_{0}
$$

with zero initial data, since $\eta G=0$. Also, $\tilde{v}=v$ for $|x|<2$, and $\tilde{G}(s, y)=0$ if $|y|>4$. So by (6.4) we have

$$
\begin{aligned}
\left\|v^{\prime}\right\|_{L^{2}\left([0, t] \times \mathbb{R}^{3} \backslash \mathcal{K}:|x|<2\right)} & =\left\|\tilde{v}^{\prime}\right\|_{L^{2}\left([0, t] \times \mathbb{R}^{3} \backslash \mathcal{K}:|x|<2\right)} \\
\leq & \leq C\|\square \tilde{v}\|_{L^{2}\left([0, t] \times \mathbb{R}^{3} \backslash \mathcal{K}\right)} \\
\leq & \leq\left\|v_{0}^{\prime}\right\|_{L^{2}\left([0, t] \times \mathbb{R}^{3} \backslash \mathcal{K}:|x|<4\right)} \\
& \quad+C\left\|v_{0}\right\|_{L^{2}\left([0, t] \times \mathbb{R}^{3} \backslash \mathcal{K}:|x|<4\right)} .
\end{aligned}
$$

One now gets (6.2) for this remaining case by applying (3.2), since $\square v_{0}=G$.

We also shall need $L_{t}^{2} L_{x}^{2}$ estimates involving the scaling and Euclidean rotation vector fields. 
Proposition 6.2. Let $v$ and $\mathcal{K}$ be as in Proposition 6.1. Then if $N$ is fixed, there is a constant $C$ so that

$$
\begin{aligned}
& \sum_{\substack{|\alpha|+m \leq N \\
m \leq 1}}\left\|L^{m} \partial_{s, x}^{\alpha} v^{\prime}\right\|_{L^{2}\left([0, t] \times \mathbb{R}^{3} \backslash \mathcal{K}:|x|<2\right)} \\
& \leq C \int_{0}^{t} \sum_{\substack{|\alpha|+m \leq N \\
m \leq 1}}\left\|\square L^{m} \partial_{s, x}^{\alpha} v(s, \cdot)\right\|_{L^{2}\left(\mathbb{R}^{3} \backslash \mathcal{K}\right)} d s \\
& \quad+C \sum_{\substack{|\alpha|+m \leq N-1 \\
m \leq 1}}\left\|\square L^{m} \partial_{s, x}^{\alpha} v\right\|_{L^{2}\left([0, t] \times \mathbb{R}^{3} \backslash \mathcal{K}\right)} .
\end{aligned}
$$

Additionally,

$$
\begin{aligned}
& \sum_{\substack{|\alpha|+|\gamma|+m \leq N \\
m \leq 1}}\left\|L^{m} \Omega^{\gamma} \partial_{s, x}^{\alpha} v^{\prime}\right\|_{L^{2}\left([0, t] \times \mathbb{R}^{3} \backslash \mathcal{K}:|x|<2\right)} \\
& \quad \leq C \int_{0}^{t} \sum_{\substack{|\alpha|+|\gamma|+m \leq N \\
m \leq 1}}\left\|\square L^{m} \Omega^{\gamma} \partial_{s, x}^{\alpha} v(s, \cdot)\right\|_{L^{2}\left(\mathbb{R}^{3} \backslash \mathcal{K}\right)} d s \\
& +C \sum_{|\alpha|+|\gamma|+m \leq N-1}\left\|\square L^{m} \Omega^{\gamma} \partial_{s, x}^{\alpha} v\right\|_{L^{2}\left([0, t] \times \mathbb{R}^{3} \backslash \mathcal{K}\right)} .
\end{aligned}
$$

Proof. We first notice that (6.5) implies (6.6) since

$$
\begin{aligned}
\sum_{\substack{|\alpha|+|\gamma|+m \leq N \\
m \leq 1}}\left\|L^{m} \Omega^{\gamma} \partial_{s, x}^{\alpha} v^{\prime}\right\|_{L^{2}\left([0, t] \times \mathbb{R}^{3} \backslash \mathcal{K}:|x|<2\right)} & \\
& \leq C \sum_{\substack{|\alpha|+m \leq N \\
m \leq 1}}\left\|L^{m} \partial_{s, x}^{\alpha} v^{\prime}\right\|_{L^{2}\left([0, t] \times \mathbb{R}^{3} \backslash \mathcal{K}:|x|<2\right)} .
\end{aligned}
$$

To prove (6.5), one repeats the proof of Proposition 6.1 using (4.11) in place of (4.7).

As in [11], one can use these estimates and the estimates for the non-obstacle case to obtain the following.

Theorem 6.3. Let $v$ and $\mathcal{K}$ be as in Proposition (6.1). Then if $N$ is fixed, there is a constant $C$ so that

$$
\begin{gathered}
(\ln (2+t))^{-1 / 2} \sum_{|\alpha| \leq N}\left\|\langle x\rangle^{-1 / 2} \partial_{s, x}^{\alpha} v^{\prime}\right\|_{L^{2}\left([0, t] \times \mathbb{R}^{3} \backslash \mathcal{K}\right)} \\
+\sum_{|\alpha| \leq N}\left\|\langle x\rangle^{-1} \partial_{s, x}^{\alpha} v\right\|_{L_{s}^{2} L_{x}^{6}\left([0, t] \times \mathbb{R}^{3} \backslash \mathcal{K}\right)} \\
\leq C \int_{0}^{t} \sum_{|\alpha| \leq N}\left\|\square \partial_{s, x}^{\alpha} v(s, \cdot)\right\|_{L^{2}\left(\mathbb{R}^{3} \backslash \mathcal{K}\right)} d s+C \sum_{|\alpha| \leq N-1}\left\|\square \partial_{s, x}^{\alpha} v\right\|_{L^{2}\left([0, t] \times \mathbb{R}^{3} \backslash \mathcal{K}\right)} .
\end{gathered}
$$


Additionally,

$$
\begin{gathered}
(\ln (2+t))^{-1 / 2} \sum_{\substack{|\alpha|+m \leq N \\
m \leq 1}}\left\|\langle x\rangle^{-1 / 2} L^{m} \partial_{s, x}^{\alpha} v^{\prime}\right\|_{L^{2}\left([0, t] \times \mathbb{R}^{3} \backslash \mathcal{K}\right)} \\
+\sum_{\substack{|\alpha|+m \leq N \\
m \leq 1}}\left\|\langle x\rangle^{-1} L^{m} \partial_{s, x}^{\alpha} v\right\|_{L_{s}^{2} L_{x}^{6}\left([0, t] \times \mathbb{R}^{3} \backslash \mathcal{K}\right)} \\
\leq C \int_{0}^{t} \sum_{\substack{|\alpha|+m \leq N \\
m \leq 1}}\left\|\square L^{m} \partial_{s, x}^{\alpha} v(s, \cdot)\right\|_{L^{2}\left(\mathbb{R}^{3} \backslash \mathcal{K}\right)} d s \\
+\sum_{\substack{|\alpha|+m \leq N-1 \\
m \leq 1}}\left\|\square L^{m} \partial_{s, x}^{\alpha} v\right\|_{L^{2}\left([0, t] \times \mathbb{R}^{3} \backslash \mathcal{K}\right)},
\end{gathered}
$$

and

$$
\begin{gathered}
(\ln (2+t))^{-1 / 2} \sum_{\substack{|\alpha|+m \leq N \\
m \leq 1}}\left\|\langle x\rangle^{-1 / 2} L^{m} Z^{\alpha} v^{\prime}\right\|_{L^{2}\left([0, t] \times \mathbb{R}^{3} \backslash \mathcal{K}\right)} \\
+\sum_{\substack{|\alpha|+m \leq N \\
m \leq 1}}\left\|\langle x\rangle^{-1} L^{m} Z^{\alpha} v\right\|_{L_{s}^{2} L_{x}^{6}\left([0, t] \times \mathbb{R}^{3} \backslash \mathcal{K}\right)} \\
\leq C \int_{0}^{t} \sum_{\substack{|\alpha|+m \leq N \\
m \leq 1}}\left\|\square L^{m} Z^{\alpha} v(s, \cdot)\right\|_{L^{2}\left(\mathbb{R}^{3} \backslash \mathcal{K}\right)} d s \\
+C \sum_{\substack{|\alpha|+m \leq N-1 \\
m \leq 1}}\left\|\square L^{m} Z^{\alpha} v\right\|_{L^{2}\left([0, t] \times \mathbb{R}^{3} \backslash \mathcal{K}\right)} .
\end{gathered}
$$

Proof. Let us first handle (6.7) since it is the simplest. In view of Proposition 6.1 and Sobolev embedding it suffices to prove that

$$
\begin{aligned}
& (\ln (2+t))^{-1 / 2} \sum_{|\alpha| \leq N}\left\|\langle x\rangle^{-1 / 2} \partial_{s, x}^{\alpha} v^{\prime}\right\|_{L^{2}\left([0, t] \times \mathbb{R}^{3} \backslash \mathcal{K}:|x|>2\right)} \\
& +\sum_{|\alpha| \leq N}\left\|\langle x\rangle^{-1} \partial_{s, x}^{\alpha} v\right\|_{L_{s}^{2} L_{x}^{6}\left([0, t] \times \mathbb{R}^{3} \backslash \mathcal{K}:|x|>2\right)} \\
& \leq C \int_{0}^{t} \sum_{|\alpha| \leq N}\left\|\square \partial_{s, x}^{\alpha} v(s, \cdot)\right\|_{L^{2}\left(\mathbb{R}^{3} \backslash \mathcal{K}\right)} d s+C \sum_{|\alpha| \leq N-1}\left\|\square \partial_{s, x}^{\alpha} v\right\|_{L^{2}\left([0, t] \times \mathbb{R}^{3} \backslash \mathcal{K}\right)} .
\end{aligned}
$$

Let us estimate the first term in the left side. For this we fix $\beta \in C^{\infty}\left(\mathbb{R}^{3}\right)$ satisfying $\beta(x)=1,|x| \geq 2$ and $\beta(x)=0,|x| \leq 3 / 2$. By assumption the obstacle is contained in the set $|x|<1$. It follows that $w=\beta v$ solves the boundaryless wave equation

$$
\square w=\beta \square v-2 \nabla_{x} \beta \cdot \nabla_{x} v-(\Delta \beta) v
$$


with zero initial data, and satisfies $w(t, x)=v(t, x),|x| \geq 2$. We split $w=w_{1}+w_{2}$, where $\square w_{1}=\beta \square v$ and $\square w_{2}=-2 \nabla_{x} \beta \cdot \nabla_{x} v-(\Delta \beta) v$. Note that by (3.1]) we have

$$
\begin{gathered}
\sum_{|\alpha| \leq N}(\ln (2+t))^{-1 / 2}\left\|\langle x\rangle^{-1 / 2} \partial_{s, x}^{\alpha} w_{1}^{\prime}\right\|_{L^{2}\left([0, t] \times \mathbb{R}^{3}\right)} \\
\leq C \sum_{|\alpha| \leq N} \int_{0}^{t}\left\|\partial_{s, x}^{\alpha} \square v(s, \cdot)\right\|_{L^{2}\left(\mathbb{R}^{3} \backslash \mathcal{K}\right)} d s,
\end{gathered}
$$

where we have used the fact that

$$
\sum_{|\alpha| \leq N} \int_{0}^{t}\left\|\partial_{s, x}^{\alpha}(\beta \square v(s, \cdot))\right\|_{L^{2}\left(\mathbb{R}^{3} \backslash \mathcal{K}\right)} d s \leq C \sum_{|\alpha| \leq N} \int_{0}^{t}\left\|\partial_{s, x}^{\alpha} \square v(s, \cdot)\right\|_{L^{2}\left(\mathbb{R}^{3} \backslash \mathcal{K}\right)} d s .
$$

To bound the first term on the left of $(\underline{6.10)}$ it therefore suffices to prove that

$$
\begin{aligned}
& (\ln (2+t))^{-1 / 2} \sum_{|\alpha| \leq N}\left\|\langle x\rangle^{-1 / 2} \partial_{s, x}^{\alpha} w_{2}^{\prime}\right\|_{L^{2}\left([0, t] \times \mathbb{R}^{3}:|x|>2\right)} \\
\leq & C \sum_{|\alpha| \leq N} \int_{0}^{t}\left\|\square \partial_{s, x}^{\alpha} v(s, \cdot)\right\|_{L^{2}\left(\mathbb{R}^{3} \backslash \mathcal{K}\right)} d s+C \sum_{|\alpha| \leq N-1}\left\|\square \partial_{s, x}^{\alpha} v\right\|_{L^{2}\left([0, t] \times \mathbb{R}^{3} \backslash \mathcal{K}\right)} .
\end{aligned}
$$

To prove (6.11) we note that $G=-2 \nabla_{x} \beta \cdot \nabla_{x} v-(\Delta \beta) v=\square w_{2}$ vanishes unless $1<|x|<2$. To use this, fix $\chi \in C_{0}^{\infty}(\mathbb{R})$ satisfying $\chi(s)=0,|s|>2$, and $\sum_{j} \chi(s-j)=1$. We then split $G=\sum_{j} G_{j}$, where $G_{j}(s, x)=\chi(s-j) G(s, x)$, and let $w_{2, j}$ be the solution of the inhomogeneous wave equation $\square w_{2, j}=G_{j}$ on Minkowski space with zero initial data. By the sharp Huygens principle, the functions $w_{2, j}$ have finite overlap, so that we have $\left|\partial_{t, x}^{\alpha} w_{2}(t, x)\right|^{2} \leq C \sum_{j}\left|\partial_{t, x}^{\alpha} w_{2, j}(t, x)\right|^{2}$, for some uniform constant $C$. Therefore, by (3.1) it follows that the square of the left side of (6.11) is dominated by

$$
\begin{aligned}
\sum_{|\alpha| \leq N} & \sum_{j}\left(\int_{0}^{t}\left\|\partial_{s, x}^{\alpha} G_{j}(s, \cdot)\right\|_{L^{2}\left(\mathbb{R}^{3}\right)} d s\right)^{2} \\
& \leq C \sum_{|\alpha| \leq N}\left\|\partial_{s, x}^{\alpha} G\right\|_{L^{2}\left([0, t] \times \mathbb{R}^{3}\right)}^{2} \\
& \leq C \sum_{|\alpha| \leq N}\left\|\partial_{s, x}^{\alpha} v^{\prime}\right\|_{L^{2}([0, t] \times\{1<|x|<2\})}^{2}+C \sum_{|\alpha| \leq N}\left\|\partial_{s, x}^{\alpha} v\right\|_{L^{2}([0, t] \times\{1<|x|<2\})}^{2} \\
& \leq C \sum_{|\alpha| \leq N}\left\|\partial_{s, x}^{\alpha} v^{\prime}\right\|_{L^{2}\left([0, t] \times \mathbb{R}^{3} \backslash \mathcal{K}:|x|<2\right)}^{2} .
\end{aligned}
$$

Consequently, the bound (6.11) follows from (6.3). Since the second term in (6.10) can also be handled by this argument, this completes the proof of (6.7). Inequalities (6.8) and (6.9) follow by a similar argument, using (6.5) and (6.6) instead of (6.3).

\section{Fixed-time $L^{2}$ estimates inVolving \\ ARBITRARY DIFFERENTIAL OPERATORS OUTSIDE OBSTACLES}

In this section we work with differential operators $P=P(t, x, D)$ that are not necessarily tangent to $\partial \mathcal{K}$, but which satisfy other conditions to be specified. We shall prove rather crude $L^{2}$ estimates for $P w$ if $w$ solves the inhomogeneous Dirichlet-wave equation (5.4) with $\mathcal{K}$ a star-shaped obstacle. In our applications, 
$P$ will be a product of powers of the Euclidean translation vector fields and the Euclidean rotation vector fields $\{\Omega\}$, as well as the scaling vector field, $L$. Neither $L$ nor the fields $\{\Omega\}$ are tangent to $\partial \mathcal{K}$ (unless $\mathcal{K}$ is a ball, in which case the $\{\Omega\}$ vanish), and therefore, unlike in the boundaryless case, we cannot deduce $L^{2}$ estimates for $P w$ directly from the energy estimate using commutation properties of the vector fields and $\square_{c}$. On the other hand, the nontangential components of $L$ and $\Omega$ on $\partial \mathcal{K}$ are bounded, which leads to estimates that we can use to prove the desired existence results.

Our basic result in this context is the following. As remarked before, the differential operator $P$ can be thought of as $P=\sum_{\substack{|\alpha|+j \leq M \\ j \leq 1}} L^{j} Z^{\alpha}$.

Proposition 7.1. Suppose that $w$ solves (5.4) where the $\gamma^{I J, j k}$ are as in Theorem 5.2. Suppose further that there is an integer $M$ and a constant $C_{0}$ so that

$$
\left|(P w)^{\prime}(t, x)\right| \leq C_{0} t \sum_{|\alpha| \leq M-1}\left|\partial_{t} \partial_{t, x}^{\alpha} w^{\prime}(t, x)\right|+C_{0} \sum_{|\alpha| \leq M}\left|\partial_{t, x}^{\alpha} w^{\prime}(t, x)\right|, \quad x \in \partial \mathcal{K} .
$$

Then, if $\square_{\gamma}$ and $\square_{c}$ are as in (5.1) and (1.3), respectively,

$$
\begin{aligned}
\left\|(P w)^{\prime}(t, \cdot)\right\|_{L^{2}\left(\mathbb{R}^{3} \backslash \mathcal{K}\right)} & \leq C \int_{0}^{t}\left\|\square_{\gamma} P w(s, \cdot)\right\|_{L^{2}\left(\mathbb{R}^{3} \backslash \mathcal{K}\right)} d s \\
& +C \int_{0}^{t} \sum_{\substack{|\alpha|+j \leq M+1 \\
j \leq 1}}\left\|\square_{c} L^{j} \partial_{s, x}^{\alpha} w(s, \cdot)\right\|_{L^{2}\left(\mathbb{R}^{3} \backslash \mathcal{K}\right)} d s \\
& +C \sum_{\substack{|\alpha|+j \leq M \\
j \leq 1}}\left\|\square_{c} L^{j} \partial_{s, x}^{\alpha} w\right\|_{L^{2}\left([0, t] \times \mathbb{R}^{3} \backslash \mathcal{K}\right)} .
\end{aligned}
$$

Proof. The proof is similar to that of Theorem 5.1, except that here we must estimate the flux terms that arise by using the trace inequality and the bounds (7.1).

To be more specific, we need to use the analogue of (5.10) where $w$ is replaced by $P w$. Therefore, if we now set

$$
e_{j}=e_{j}(P w), \quad j=0,1,2,3,
$$

then (5.10) in our context becomes

$$
\begin{aligned}
& \partial_{t} \int_{\mathbb{R}^{3} \backslash \mathcal{K}} e_{0}(t, x) d x-\int_{\partial \mathcal{K}} \sum_{j=1}^{3} e_{j} n_{j} d \sigma \\
&=2 \int_{\mathbb{R}^{3} \backslash \mathcal{K}}\left\langle\partial_{t} P w, \square_{\gamma} P w\right\rangle d x+\int_{\mathbb{R}^{3} \backslash \mathcal{K}} R\left((P w)^{\prime},(P w)^{\prime}\right) d x,
\end{aligned}
$$

where as before $R$ is a quadratic form whose coefficients belong to $L_{t}^{1} L_{x}^{\infty}$. Therefore, if as in the proof of Theorem [5.1 we use (5.2) and (5.3) and apply Gronwall's inequality, we conclude that if $\delta>0$ is small enough, then

$$
\begin{aligned}
\left\|(P w)^{\prime}(t, \cdot)\right\|_{L^{2}\left(\mathbb{R}^{3} \backslash \mathcal{K}\right)} & \leq C \int_{0}^{t}\left\|\square_{\gamma} P w(s, \cdot)\right\|_{L^{2}\left(\mathbb{R}^{3} \backslash \mathcal{K}\right)} d s \\
& +C\left(\int_{[0, t] \times \partial \mathcal{K}}\left(\left|\partial_{t} P w(s, x)\right|^{2}+\left|\nabla_{x} P w(s, x)\right|^{2}\right) d \sigma\right)^{1 / 2} .
\end{aligned}
$$


Recall that we are assuming $\mathcal{K} \subset\left\{x \in \mathbb{R}^{3}:|x|<1\right\}$. Therefore, by (17.1) and a trace argument we have

$$
\left(\int_{[0, t] \times \partial \mathcal{K}}\left|(P w)^{\prime}(s, x)\right|^{2} d \sigma\right)^{1 / 2} \leq C \sum_{\substack{|\alpha|+j \leq M+1 \\ j \leq 1}}\left\|L^{j} \partial_{s, x}^{\alpha} w^{\prime}\right\|_{L^{2}\left([0, t] \times \mathbb{R}^{3} \backslash \mathcal{K}:|x|<2\right)}
$$

One therefore gets (7.2) from (6.5).

As an immediate corollary we have the following.

Corollary 7.2. Assume that $w$ solves (5.4). Then if $M=1,2, \ldots$,

$$
\begin{aligned}
& \sum_{\substack{|\alpha|+j \leq M \\
j \leq 1}}\left\|\left(L^{j} Z^{\alpha} w\right)^{\prime}(t, \cdot)\right\|_{L^{2}\left(\mathbb{R}^{3} \backslash \mathcal{K}\right)} \leq C \int_{0}^{t} \sum_{\substack{|\alpha|+j \leq M \\
j \leq 1}}\left\|\square_{\gamma} L^{j} Z^{\alpha} w(s, \cdot)\right\|_{L^{2}\left(\mathbb{R}^{3} \backslash \mathcal{K}\right)} d s \\
&+C \int_{0}^{t} \sum_{\substack{|\alpha|+j \leq M+1 \\
j \leq 1}}\left\|\square_{c} L^{j} \partial_{s, x}^{\alpha} w(s, \cdot)\right\|_{L^{2}\left(\mathbb{R}^{3} \backslash \mathcal{K}\right)} d s \\
&+C \sum_{\substack{|\alpha|+j \leq M \\
j \leq 1}}\left\|\square_{c} L^{j} \partial_{s, x}^{\alpha} w\right\|_{L^{2}\left([0, t] \times \mathbb{R}^{3} \backslash \mathcal{K}\right)} .
\end{aligned}
$$

\section{8. $L_{x}^{2}$ ESTIMATES INVOLVING ONLY THE SCALING AND TRANSLATION VECTOR FIELDS OUTSIDE STAR-SHAPED OBSTACLES}

In this section we prove $L_{x}^{2}$ estimates involving a single occurrence of the scaling vector field $L=t \partial_{t}+x \cdot \nabla_{x}$. Recall that the commutator of $L$ with $\square_{c}$ is $2 \square_{c}$. For obstacle problems, the complication arises that $L$ does not preserve Dirichlet boundary conditions. Because of this, unlike in the boundaryless setting, one cannot derive $L^{2}$ estimates for $L u$ just by using energy estimates. Fortunately, though, if one assumes that $\partial \mathcal{K}$ is star-shaped, then in the proof of the energy estimates, $L$ contributes a term with a favorable sign, as in the classical Morawetz inequality for star-shaped domains [18]. For this reason, we can estimate $L u^{\prime}$ in $L^{2}$, although there is a slight loss versus the corresponding estimates for Minkowski space. This slight loss is reflected especially in the third and fourth terms on the right of 8.3 below. Unlike the corresponding terms on the right side of (7.4), these terms involve only translation derivatives and as such are easily handled in the nonlinear applications to follow.

To prove the estimates of this section requires strengthening the hypotheses on the metric perturbations $\gamma^{I J, j k}$. We shall assume as before that (5.3) holds, but need to strengthen (5.2) to

$$
\sum_{I, J, j, k}\left|\gamma^{I J, j k}(t, x)\right| \leq \delta /(1+t)
$$

with $\delta>0$ small enough so that (5.12) holds. Under these assumptions, we have 
Proposition 8.1. Let $w$ solve (5.4) with $\gamma$ as in (5.3), 8.1). Then

$$
\begin{aligned}
\left\|(L w)^{\prime}(t, \cdot)\right\|_{L^{2}\left(\mathbb{R}^{3} \backslash \mathcal{K}\right)} & \leq C \int_{0}^{t}\left\|\square_{\gamma} L w(s, \cdot)\right\|_{L^{2}\left(\mathbb{R}^{3} \backslash \mathcal{K}\right)} d s \\
& +C \int_{0}^{t} \sum_{|\alpha| \leq 2}\left\|\square_{c} \partial_{s, x}^{\alpha} w(s, \cdot)\right\|_{L^{2}\left(\mathbb{R}^{3} \backslash \mathcal{K}\right)} d s \\
& +C \sum_{|\alpha| \leq 1}\left\|\square_{c} \partial_{t, x}^{\alpha} w\right\|_{L^{2}\left([0, t] \times \mathbb{R}^{3} \backslash \mathcal{K}\right)} .
\end{aligned}
$$

As a corollary of this and (5.12) we have the following useful estimate.

Theorem 8.2. Let $w$ solve (5.4) with $\gamma$ as in (5.3), (8.1). Then if $N=0,1,2, \ldots$ is fixed,

$$
\begin{aligned}
& \sum_{\substack{|\alpha|+m \leq N \\
m \leq 1}}\left\|L^{m} \partial_{t, x}^{\alpha} w^{\prime}(t, \cdot)\right\|_{L^{2}\left(\mathbb{R}^{3} \backslash \mathcal{K}\right)} \\
& \leq C \int_{0}^{t} \sum_{\substack{|\alpha|+m \leq N \\
m \leq 1}}\left\|\square_{\gamma} L^{m} \partial_{s, x}^{\alpha} w(s, \cdot)\right\|_{L^{2}\left(\mathbb{R}^{3} \backslash \mathcal{K}\right)} d s \\
& \quad+C \sum_{|\alpha|+m \leq N-1}\left\|\square_{c} L^{m} \partial_{t, x}^{\alpha} w(t, \cdot)\right\|_{L^{2}\left(\mathbb{R}^{3} \backslash \mathcal{K}\right)} \\
& \quad+C \int_{0}^{t} \sum_{|\alpha| \leq N+1}\left\|\square_{c} \partial_{s, x}^{\alpha} w(s, \cdot)\right\|_{L^{2}\left(\mathbb{R}^{3} \backslash \mathcal{K}\right)} d s+C \sum_{|\alpha| \leq N}\left\|\square_{c} \partial_{s, x}^{\alpha} w\right\|_{L^{2}\left([0, t] \times \mathbb{R}^{3} \backslash \mathcal{K}\right)} .
\end{aligned}
$$

The proof that Theorem 8.2 follows from Proposition 8.1 requires a simple modification of the proof that Theorem 5.2 follows from Theorem 5.1. Precisely, we first note that, if $m=0$, then Theorem 8.2 follows from Theorem 5.2 For $m=1$, we apply induction on the number of spatial derivatives in $\alpha$. The elliptic regularity estimate required in this step is that, for $N \geq 2$,

$$
\begin{array}{r}
\sum_{|\alpha|=N}\left\|\partial_{x}^{\alpha} L w\right\|_{L^{2}\left(\mathbb{R}^{3} \backslash \mathcal{K}\right)} \leq C \sum_{|\alpha| \leq N-2}\left(\left\|\partial_{x}^{\alpha} \Delta L w\right\|_{L^{2}\left(\mathbb{R}^{3} \backslash \mathcal{K}\right)}+\left\|\partial_{x}^{\alpha}(L w)^{\prime}\right\|_{L^{2}\left(\mathbb{R}^{3} \backslash \mathcal{K}\right)}\right) \\
+C\left\|\left.(L w)\right|_{\partial \mathcal{K}}\right\|_{H^{N-\frac{1}{2}(\partial \mathcal{K})}}
\end{array}
$$

This holds locally by standard elliptic regularity (see e.g. [3], Theorem 8.13), and the fact that $L w$ is locally controlled by $(L w)^{\prime}$ and the trace of $L w$. Using cutoff functions one can then reduce to the boundaryless case, where only the first term on the right is required.

Since $\left.(L w)\right|_{\partial \mathcal{K}}=\left.\left(x \cdot \partial_{x} w\right)\right|_{\partial \mathcal{K}}$, by the trace theorem we have

$$
\left\|\left.(L w)\right|_{\partial \mathcal{K}}\right\|_{H^{N-\frac{1}{2}(\partial \mathcal{K})}} \leq C \sum_{|\alpha| \leq N}\left\|\partial_{x}^{\alpha} w^{\prime}\right\|_{L^{2}\left(\mathbb{R}^{3} \backslash \mathcal{K}\right)},
$$

and the right-hand side involves the estimate (8.2) for the case $m=0$. It remains, then, to prove Proposition 8.1 
To prove (8.2), we need to use the analogue of (5.10) where $w$ there is replaced by $L w$. Therefore, if we set

$$
e_{j}=e_{j}(L w), \quad j=0,1,2,3,
$$

then (5.10) in our context becomes

$$
\begin{aligned}
& \partial_{t} \int_{\mathbb{R}^{3} \backslash \mathcal{K}} e_{0}(t, x) d x-\int_{\partial \mathcal{K}} \sum_{j=1}^{3} e_{j} n_{j} d \sigma \\
&=2 \int_{\mathbb{R}^{3} \backslash \mathcal{K}}\left\langle\partial_{t} L w, \square_{\gamma} L w\right\rangle d x+\int_{\mathbb{R}^{3} \backslash \mathcal{K}} R\left((L w)^{\prime},(L w)^{\prime}\right) d x
\end{aligned}
$$

where as before $R$ is a quadratic form whose coefficients belong to $L_{t}^{1} L_{x}^{\infty}$.

We can simplify the last term on the left-hand side. We first notice that, at points $(s, x)$ belonging to $\mathbb{R}_{+} \times \partial \mathcal{K}$, the Dirichlet boundary conditions on $w$ give us

$$
\partial_{s} L w^{I}=s \partial_{s}^{2} w^{I}+\partial_{s} w^{I}+\partial_{s}\left\langle x, \nabla_{x}\right\rangle w^{I}=\partial_{s}\left\langle x, \nabla_{x}\right\rangle w^{I}=\langle x, \vec{n}\rangle \partial_{\vec{n}} \partial_{s} w^{I},
$$

where $\partial_{\vec{n}} w^{I}=\left\langle\vec{n}, \nabla_{x}\right\rangle w^{I}$ denotes differentiation with respect to the outward normal to $\mathcal{K}$. Similarly,

$$
\sum_{j=1}^{3} n_{j} \partial_{j} L w^{I}=s \partial_{\vec{n}} \partial_{s} w^{I}+\partial_{\vec{n}}\left(\left\langle x, \nabla_{x}\right\rangle w^{I}\right)
$$

on $\mathbb{R}_{+} \times \partial \mathcal{K}$. As a consequence, we have

$$
\begin{aligned}
& -\sum_{j=1}^{3} e_{j} n_{j}=2 \sum_{I=1}^{N}\left[\langle x, \vec{n}\rangle c_{I}^{2} s\left(\partial_{\vec{n}} \partial_{s} w^{I}\right)^{2}\right. \\
& \left.+\langle x, \vec{n}\rangle c_{I}^{2} \partial_{\vec{n}} \partial_{s} w^{I} \partial_{n}\left(\left\langle x, \nabla_{x}\right\rangle w^{I}\right)-\langle x, \vec{n}\rangle \partial_{\vec{n}} \partial_{s} w^{I} \sum_{J=1}^{N} \sum_{j=1}^{3} \sum_{k=0}^{3} \gamma^{I J, j k} n_{j} \partial_{k} L w^{I}\right] .
\end{aligned}
$$

Since we are assuming (8.1), we have

$$
-\sum_{j=1}^{3} e_{j} n_{j}=2 \sum_{I=1}^{N} c_{I}^{2}\langle x, \vec{n}\rangle s\left|\partial_{\vec{n}} \partial_{s} w^{I}\right|^{2}-Q\left(w^{\prime \prime}, w^{\prime}\right),
$$

where

$$
\left|Q\left(w^{\prime \prime}, w^{\prime}\right)\right| \leq C \sum_{1 \leq|\alpha| \leq 2}\left|\partial_{s, x}^{\alpha} w\right|^{2}
$$

for some uniform constant $C$. Because of this, identity (8.4) yields

$$
\begin{aligned}
& \partial_{t} \int_{\mathbb{R}^{3} \backslash \mathcal{K}} e_{0}(t, x) d x+\int_{\partial \mathcal{K}} 2 \sum_{I=1}^{N} c_{I}^{2}\langle x, \vec{n}\rangle s\left|\partial_{\vec{n}} \partial_{s} w^{I}\right|^{2} d \sigma \\
& \quad=\int_{\partial \mathcal{K}} Q\left(w^{\prime \prime}, w^{\prime}\right) d \sigma+2 \int_{\mathbb{R}^{3} \backslash \mathcal{K}}\left\langle\partial_{t} L w, \square_{\gamma} L w\right\rangle d x+\int_{\mathbb{R}^{3} \backslash \mathcal{K}} R\left((L w)^{\prime},(L w)^{\prime}\right) d x
\end{aligned}
$$


The second term on the left-hand side is positive, since $\langle x, \vec{n}\rangle>0$ for star-shaped $\mathcal{K}$. Hence, we can apply Gronwall's inequality to obtain

$$
\begin{aligned}
\left\|(L w)^{\prime}(t, \cdot)\right\|_{L^{2}\left(\mathbb{R}^{3} \backslash \mathcal{K}\right)} \leq C \int_{0}^{t}\left\|\square_{\gamma} L w(s, \cdot)\right\|_{L^{2}\left(\mathbb{R}^{3} \backslash \mathcal{K}\right)} d s & \\
& +C\left(\sum_{1 \leq|\alpha| \leq 2} \int_{[0, t] \times \partial \mathcal{K}}\left|\partial_{s, x}^{\alpha} w\right|^{2} d \sigma\right)^{1 / 2} .
\end{aligned}
$$

The first term on the right here is contained in the right side of (8.2). As a result, it suffices to show that the last term in the preceding inequality is dominated by the other terms in the right side of (8.2). But

$$
\left(\sum_{1 \leq|\alpha| \leq 2} \int_{[0, t] \times \partial \mathcal{K}}\left|\partial_{s, x}^{\alpha} w\right|^{2} d \sigma\right)^{1 / 2} \leq C \sum_{|\alpha| \leq 2}\left\|\partial_{s, x}^{\alpha} w^{\prime}(s, \cdot)\right\|_{L^{2}\left([0, t] \times \mathbb{R}^{3} \backslash \mathcal{K}:|x|<2\right)},
$$

so that (6.3) yields the desired bounds for this term as well. This completes the proof of (8.2), and hence Proposition 8.1

\section{MAin $L^{2}$ ESTimates OUtSide STAR-Shaped OBstaCles}

We shall assume here that $\mathcal{K} \subset \mathbb{R}^{3}$ is star-shaped. We shall also assume that the $\gamma^{I J, j k}$ satisfy (5.3) and (8.1). Then if we combine our $L^{2}$ estimates we have the following useful result.

Theorem 9.1. Let $w \in C^{\infty}$ solve (5.4) and vanish for $t<0$. Suppose also that $\mathcal{K}$ is star-shaped (see (1.1)) and the $\gamma^{I J, j k}$ are as in (5.3), (8.1). Then if $N=0,1,2, \ldots$ is fixed, we have

$$
\begin{aligned}
\sum_{|\alpha| \leq N+4}\left\|\partial_{t, x}^{\alpha} w^{\prime}(t, \cdot)\right\|_{L^{2}\left(\mathbb{R}^{3} \backslash \mathcal{K}\right)}+\sum_{\substack{|\alpha|+m \leq N+2 \\
m \leq 1}}\left\|L^{m} \partial_{t, x}^{\alpha} w^{\prime}(t, \cdot)\right\|_{L^{2}\left(\mathbb{R}^{3} \backslash \mathcal{K}\right)} \\
+\sum_{\substack{|\alpha|+m \leq N \\
m \leq 1}}\left\|L^{m} Z^{\alpha} w^{\prime}(t, \cdot)\right\|_{L^{2}\left(\mathbb{R}^{3} \backslash \mathcal{K}\right)} \\
\leq C \int_{0}^{t}\left(\sum_{|\alpha| \leq N+4}\left\|\square_{\gamma} \partial_{s, x}^{\alpha} w(s, \cdot)\right\|_{L^{2}\left(\mathbb{R}^{3} \backslash \mathcal{K}\right)}\right. \\
+\sum_{\substack{|\alpha|+m \leq N+2 \\
m \leq 1}}\left\|\square_{\gamma} L^{m} \partial_{s, x}^{\alpha} w(s, \cdot)\right\|_{L^{2}\left(\mathbb{R}^{3} \backslash \mathcal{K}\right)} \\
\left.+\sum_{\substack{|\alpha|+m \leq N \\
m \leq 1}}\left\|\square_{\gamma} L^{m} Z^{\alpha} w(s, \cdot)\right\|_{L^{2}\left(\mathbb{R}^{3} \backslash \mathcal{K}\right)}\right) d s
\end{aligned}
$$




$$
\begin{aligned}
& +C \sum_{|\alpha| \leq N+3}\left\|\square_{\gamma} \partial_{t, x}^{\alpha} w(t, \cdot)\right\|_{L^{2}\left(\mathbb{R}^{3} \backslash \mathcal{K}\right)} \\
& +C \sum_{\substack{|\alpha|+m \leq N+1 \\
m \leq 1}}\left\|\square_{\gamma} L^{m} \partial_{t, x}^{\alpha} w(t, \cdot)\right\|_{L^{2}\left(\mathbb{R}^{3} \backslash \mathcal{K}\right)} \\
& +C \sum_{|\alpha| \leq N+2}\left\|\square_{c} \partial_{s, x}^{\alpha} w\right\|_{L^{2}\left([0, t] \times \mathbb{R}^{3} \backslash \mathcal{K}\right)} \\
& \quad+C \sum_{\substack{|\alpha|+m \leq N \\
m \leq 1}}\left\|\square_{C} L^{m} \partial_{s, x}^{\alpha} w\right\|_{L^{2}\left([0, t] \times \mathbb{R}^{3} \backslash \mathcal{K}\right)} .
\end{aligned}
$$

To see this, let the left side be denoted by $I+I I+I I I$, and let $R H S$ denote the right side. We then claim that

$$
\begin{aligned}
& I \leq R H S+\sum_{I, J, j, k} \sum_{|\alpha| \leq N+3}\left\|\gamma^{I J, j k} \partial_{j} \partial_{k} \partial_{t, x}^{\alpha} w(t, \cdot)\right\|_{L^{2}\left(\mathbb{R}^{3} \backslash \mathcal{K}\right)} \\
& I I \leq R H S+C \int_{0}^{t} \sum_{|\alpha| \leq N+3} \sum_{I, J, j, k}\left\|\gamma^{I J, j k} \partial_{j} \partial_{k} \partial_{s, x}^{\alpha} w(s, \cdot)\right\|_{L^{2}\left(\mathbb{R}^{3} \backslash \mathcal{K}\right)} d s \\
& \quad+\sum_{\substack{|\alpha|+m \leq N+1 \\
m \leq 1}} \sum_{\substack{m, J, j, k \\
m \leq}}\left\|\gamma^{I J, j k} \partial_{j} \partial_{k} L^{m} \partial_{t, x}^{\alpha} w(t, \cdot)\right\|_{L^{2}\left(\mathbb{R}^{3} \backslash \mathcal{K}\right)} \\
& \int_{0}^{t} \sum_{\substack{|\alpha|+m \leq N+1 \\
m \leq 1}} \sum_{I, J, j, k}\left\|\gamma^{I J, j k} \partial_{j} \partial_{k} L^{m} \partial_{s, x}^{\alpha} w(s, \cdot)\right\|_{L^{2}\left(\mathbb{R}^{3} \backslash \mathcal{K}\right)} d s .
\end{aligned}
$$

Indeed, by (5.12), $I$ is dominated by the first and fourth terms in the right side of (9.1) along with the last term in (9.2). Also, by (8.3), II is dominated by the second, fifth, first, and sixth terms in the right side of (9.1) along with the last two terms in (9.3). Lastly, by (7.4), III is dominated by the third, second, and seventh terms in the right side of (8.2), along with the last term in (9.4). By inequality (5.2), if $\delta$ is sufficiently small, we can absorb the time $t$ terms on the right-hand side of (9.2) and (9.3) into the left-hand side of (9.1). The inequality (9.1) now follows from (5.3) and (9.2)-(9.4) by Gronwall's inequality.

Repeating this proof and using Theorem 6.3 yields the following result, which will be used in the iteration argument of the next section.

Corollary 9.2. Let $w, \mathcal{K}$ and $\gamma^{I J, j k}$ be as in Theorem 9.1, Then if $N$ is fixed,

$$
\begin{gathered}
\sum_{|\alpha| \leq N+4}\left\|\partial_{t, x}^{\alpha} w^{\prime}(t, \cdot)\right\|_{L^{2}\left(\mathbb{R}^{3} \backslash \mathcal{K}\right)}+\sum_{\substack{|\alpha|+m \leq N+2 \\
m \leq 1}}\left\|L^{m} \partial_{t, x}^{\alpha} w^{\prime}(t, \cdot)\right\|_{L^{2}\left(\mathbb{R}^{3} \backslash \mathcal{K}\right)} \\
+\sum_{\substack{|\alpha|+m \leq N \\
m \leq 1}}\left\|L^{m} Z^{\alpha} w^{\prime}(t, \cdot)\right\|_{L^{2}\left(\mathbb{R}^{3} \backslash \mathcal{K}\right)}
\end{gathered}
$$




$$
\begin{aligned}
& +(\ln (2+t))^{-1 / 2}\left(\sum_{|\alpha| \leq N+3}\left\|\langle x\rangle^{-1 / 2} \partial_{s, x}^{\alpha} w^{\prime}\right\|_{L^{2}\left([0, t] \times \mathbb{R}^{3} \backslash \mathcal{K}\right)}\right. \\
& +\sum_{\substack{|\alpha|+m \leq N+1 \\
m \leq 1}}\left\|\langle x\rangle^{-1 / 2} L^{m} \partial_{s, x}^{\alpha} w^{\prime}\right\|_{L^{2}\left([0, t] \times \mathbb{R}^{3} \backslash \mathcal{K}\right)} \\
& \left.+\sum_{\substack{|\alpha|+m \leq N-1 \\
m \leq 1}}\left\|\langle x\rangle^{-1 / 2} L^{m} Z^{\alpha} w^{\prime}\right\|_{L^{2}\left([0, t] \times \mathbb{R}^{3} \backslash \mathcal{K}\right)}\right) \\
& +\left(\sum_{|\alpha| \leq N+3}\left\|\langle x\rangle^{-1} \partial_{s, x}^{\alpha} w\right\|_{L_{s}^{2} L_{x}^{6}\left([0, t] \times \mathbb{R}^{3} \backslash \mathcal{K}\right)}\right. \\
& +\sum_{\substack{|\alpha|+m \leq N+1 \\
m \leq 1}}\left\|\langle x\rangle^{-1} L^{m} \partial_{s, x}^{\alpha} w\right\|_{L_{s}^{2} L_{x}^{6}\left([0, t] \times \mathbb{R}^{3} \backslash \mathcal{K}\right)} \\
& \left.+\sum_{\substack{|\alpha|+m \leq N-1 \\
m \leq 1}}\left\|\langle x\rangle^{-1} L^{m} Z^{\alpha} w\right\|_{L_{s}^{2} L_{x}^{6}\left([0, t] \times \mathbb{R}^{3} \backslash \mathcal{K}\right)}\right) \\
& \leq C \int_{0}^{t}\left(\sum_{|\alpha| \leq N+4}\left\|\square_{\gamma} \partial_{s, x}^{\alpha} w(s, \cdot)\right\|_{L^{2}\left(\mathbb{R}^{3} \backslash \mathcal{K}\right)}+\sum_{\substack{|\alpha|+m \leq N+2 \\
m \leq 1}}\left\|\square_{\gamma} L^{m} \partial_{s, x}^{\alpha} w(s, \cdot)\right\|_{L^{2}\left(\mathbb{R}^{3} \backslash \mathcal{K}\right)}\right. \\
& \left.+\sum_{\substack{|\alpha|+m \leq N \\
m \leq 1}}\left\|\square_{\gamma} L^{m} Z^{\alpha} w(s, \cdot)\right\|_{L^{2}\left(\mathbb{R}^{3} \backslash \mathcal{K}\right)}\right) d s \\
& +C \sum_{|\alpha| \leq N+3}\left\|\square_{\gamma} \partial_{t, x}^{\alpha} w(t, \cdot)\right\|_{L^{2}\left(\mathbb{R}^{3} \backslash \mathcal{K}\right)} \\
& +C \sum_{\substack{|\alpha|+m \leq N+1 \\
m \leq 1}}\left\|\square_{\gamma} L^{m} \partial_{t, x}^{\alpha} w(t, \cdot)\right\|_{L^{2}\left(\mathbb{R}^{3} \backslash \mathcal{K}\right)} \\
& +C \sum_{|\alpha| \leq N+2}\left\|\square_{c} \partial_{s, x}^{\alpha} w\right\|_{L^{2}\left([0, t] \times \mathbb{R}^{3} \backslash \mathcal{K}\right)} \\
& +C \sum_{\substack{|\alpha|+m \leq N \\
m \leq 1}}\left\|\square_{c} L^{m} \partial_{s, x}^{\alpha} w\right\|_{L^{2}\left([0, t] \times \mathbb{R}^{3} \backslash \mathcal{K}\right)} \\
& +C \sum_{\substack{|\alpha|+m \leq N-2 \\
m \leq 1}}\left\|\square_{c} L^{m} Z^{\alpha} w\right\|_{L^{2}\left([0, t] \times \mathbb{R}^{3} \backslash \mathcal{K}\right)} .
\end{aligned}
$$

10. Almost global existence for quasilinear wave equations OUTSIDE OF STAR-SHAPED OBSTACLES

We conclude by showing how to adapt the proof of Theorem 1.2 to establish almost global existence for the system (1.2). As in [10, [11, it is convenient to reduce the Cauchy problem (1.2) to an equivalent equation with driving force but vanishing Cauchy data, in order to avoid dealing with compatibility conditions for the Cauchy data. We can then set up an iteration argument for the new equation similar to that used in the proof of Theorem 1.2 . 
As in the boundaryless case, we do not need to assume that the data has compact support. Here, however, we have to replace the smallness condition (3.12) by

$$
\sum_{|\alpha| \leq 15}\left\|\langle x\rangle^{|\alpha|} \partial_{x}^{\alpha} f\right\|_{L^{2}\left(\mathbb{R}^{3} \backslash \mathcal{K}\right)}+\sum_{|\alpha| \leq 14}\left\|\langle x\rangle^{|\alpha|+1} \partial_{x}^{\alpha} g\right\|_{L^{2}\left(\mathbb{R}^{3} \backslash \mathcal{K}\right)} \leq \varepsilon .
$$

The extra number of derivatives required is due to the loss of four derivatives in the $L^{2}$ estimates for the obstacle case versus the non-obstacle case. The extra power of $\langle x\rangle$ and our assumption here that we control the size of $(f, g)$ as well as their derivatives, are used in the steps following (10.8) below.

To make the reduction to an equation with zero initial data, we first note that if the data satisfy (10.1) with $\varepsilon>0$ small, then we can construct the solution $u$ to the system (1.2) on the set $(t, x) \in\{0<c t<|x|\} \cap\left\{\left[0, T_{\varepsilon}\right) \times \mathbb{R}^{3} \backslash \mathcal{K}\right\}$, where

$$
c=5 \max _{I} c_{I}
$$

and that on this set the solution satisfies

$$
\sup _{0 \leq t \leq \infty} \sum_{|\alpha| \leq 15}\left\|\langle x\rangle^{|\alpha|} \partial_{t, x}^{\alpha} u(t, \cdot)\right\|_{L^{2}\left(\mathbb{R}^{3} \backslash \mathcal{K}:|x|>c t\right)} \leq C_{0} \varepsilon .
$$

To see this, we note that by scaling the $t$ variable we may assume that $\max _{I} c_{I}=\frac{1}{2}$. The local existence results in [10] yield a solution $u$ to (1.2) on the set $(t, x) \in[0,2] \times$ $\mathbb{R}^{3} \backslash \mathcal{K}$, satisfying the bounds 10.3 To see that this solution can be extended to include all $(t, x)$ with $0<c t<|x|$, we let $R \geq 4$ and consider data $\left(f_{R}, g_{R}\right)$ supported in $R / 4<|x|<4 R$, which agrees with the data $(f, g)$ on the set $R / 2<$ $|x|<2 R$. Let $u_{R}(t, x)$ satisfy the boundaryless equation

$$
\square_{c} u_{R}=Q\left(d u_{R}, R^{-1} d^{2} u_{R}\right),
$$

with Cauchy data $\left(f_{R}(R \cdot), R g_{R}(R \cdot)\right)$. (Recall that $Q$ is the nonlinearity appearing in the equation, see (1.2), (1.4).) Because of our smallness assumption (1.7) on $(f, g)$, the solution $u_{R}$ of (10.4) exists for $0<t<1$ by standard results (see e.g. 6]), and satisfies

$$
\begin{aligned}
& \sup _{0 \leq t \leq 1}\left\|u_{R}(t, \cdot)\right\|_{H^{15}\left(\mathbb{R}^{3}\right)} \leq C\left(\left\|f_{R}(R \cdot)\right\|_{H^{15}\left(\mathbb{R}^{3}\right)}+R\left\|g_{R}(R \cdot)\right\|_{H^{14}\left(\mathbb{R}^{3}\right)}\right) \\
& \leq C R^{-3 / 2}\left(\sum_{|\alpha| \leq 15}\left\|\left(R \partial_{x}\right)^{\alpha} f_{R}\right\|_{L^{2}\left(\mathbb{R}^{3}\right)}+R \sum_{|\alpha| \leq 14}\left\|\left(R \partial_{x}\right)^{\alpha} g_{R}\right\|_{L^{2}\left(\mathbb{R}^{3}\right)}\right) .
\end{aligned}
$$

The smallness condition on $\left|u_{R}^{\prime}\right|$ implies that the wave speeds for the quasilinear equation (10.4) are bounded above by 1 . A domain of dependence argument shows that the solutions $u_{R}\left(R^{-1} t, R^{-1} x\right)$ restricted to ||$x|-R|<\frac{R}{2}-t$ agree on their overlaps, and also with the local solution $u$, yielding the solution to (1.2) on the desired set $(t, x) \in\{0<c t<|x|\} \cap\left\{\left[0, T_{\varepsilon}\right) \times \mathbb{R}^{3} \backslash \mathcal{K}\right\}$. A partition of unity argument now yields (10.3).

We use this partial construction of the solution $u$ to start our iteration. Fix a cutoff function $\chi \in C^{\infty}(\mathbb{R})$ satisfying $\chi(s)=1$ if $s \leq \frac{1}{2 c}$ and $\chi(s)=0$ if $s>\frac{1}{c}$, with $c$ as in (10.2). Set

$$
u_{0}(t, x)=\eta(t, x) u(t, x), \quad \eta(t, x)=\chi\left(|x|^{-1} t\right) .
$$

\footnotetext{
${ }^{2}$ The local existence theorem in [10] was stated only for diagonal systems. However, since the proof was based only on energy estimates, it also applies to nonlinear systems that satisfy the symmetry condition 1.5 , using Theorem 5.1
} 
Note that since $|x|$ is bounded below on the complement of $\mathcal{K}$, the function $\eta(t, x)$ is smooth and homogeneous of degree 0 on $(t, x) \in\left[0, T_{\varepsilon}\right) \times\left(\mathbb{R}^{3} \backslash \mathcal{K}\right)$. Also,

$$
\square_{c} u_{0}=\eta Q\left(d u, d^{2} u\right)+\left[\square_{c}, \eta\right] u .
$$

Thus, $u$ solves $\square_{c} u=Q\left(d u, d^{2} u\right)$ for $0<t<T_{\varepsilon}$ and $x \in \mathbb{R}^{3} \backslash \mathcal{K}$ if and only if $w=u-u_{0}$ solves

$$
\left\{\begin{array}{l}
\square_{c} w=(1-\eta) Q\left(d\left(u_{0}+w\right), d^{2}\left(u_{0}+w\right)\right)-\left[\square_{c}, \eta\right] u, \\
\left.w\right|_{\partial \mathcal{K}}=0, \\
w(t, x)=0, \quad t \leq 0
\end{array}\right.
$$

for $0<t<T_{\varepsilon}$. We emphasize that $u_{0}$ has been constructed for all $(t, x) \in\left[0, T_{\varepsilon}\right) \times$ $\left(\mathbb{R}^{3} \backslash \mathcal{K}\right)$, and the solution $u$ has been constructed on the support of $\left[\square_{c}, \eta\right]$, so that (10.5) should be viewed as a nonlinear problem for $w$.

We shall solve (10.5) by iteration. We set $w_{0}=0$, and recursively define $w_{k}$ for $k=1,2, \ldots$ by requiring that

$$
\left\{\begin{array}{l}
\square_{c} w_{k}=(1-\eta) Q\left(d\left(u_{0}+w_{k-1}\right), d^{2}\left(u_{0}+w_{k}\right)\right)-\left[\square_{c}, \eta\right] u, \\
\left.w_{k}\right|_{\partial \mathcal{K}}=0, \\
w_{k}(t, x)=0, \quad t \leq 0 .
\end{array}\right.
$$

In place of (3.15), we now let

$$
\begin{gathered}
M_{k}(T)=\sup _{0 \leq t \leq T}\left(\sum_{|\alpha| \leq 14}\left\|\partial_{t, x}^{\alpha} w_{k}^{\prime}(t, \cdot)\right\|_{L^{2}\left(\mathbb{R}^{3} \backslash \mathcal{K}\right)}+\sum_{\substack{|\alpha|+m \leq 12 \\
m \leq 1}}\left\|L^{m} \partial_{t, x}^{\alpha} w_{k}^{\prime}(t, \cdot)\right\|_{L^{2}\left(\mathbb{R}^{3} \backslash \mathcal{K}\right)}\right. \\
\left.+\sum_{\substack{|\alpha|+m \leq 10 \\
m \leq 1}}\left\|L^{m} Z^{\alpha} w_{k}^{\prime}(t, \cdot)\right\|_{L^{2}\left(\mathbb{R}^{3} \backslash \mathcal{K}\right)}+(1+t) \sum_{|\alpha| \leq 2}\left\|Z^{\alpha} w_{k}(t, \cdot)\right\|_{L^{\infty}\left(\mathbb{R}^{3} \backslash \mathcal{K}\right)}\right) \\
+\left(\sum_{|\alpha| \leq 13} \mid\left\|\langle x\rangle^{-1 / 2} \partial_{s, x}^{\alpha} w_{k}^{\prime}\right\|_{L^{2}\left([0, T] \times \mathbb{R}^{3} \backslash \mathcal{K}\right)}\right. \\
+\sum_{|\alpha|+m \leq 11}\left\|\langle x\rangle^{-1 / 2} L^{m} \partial_{s, x}^{\alpha} w_{k}^{\prime}\right\|_{L^{2}\left([0, T] \times \mathbb{R}^{3} \backslash \mathcal{K}\right)} \\
\left.+\sum_{\substack{|\alpha|+m \leq 1 \\
m \leq 1}}\left\|\langle x\rangle^{-1 / 2} L^{m} Z^{\alpha} w_{k}^{\prime}\right\|_{L^{2}\left([0, T] \times \mathbb{R}^{3} \backslash \mathcal{K}\right)}\right) \\
+\sum_{|\alpha| \leq 13}\left\|\langle x\rangle^{-1} \partial_{s, x}^{\alpha} w_{k}\right\|_{L_{s}^{2} L_{x}^{6}\left([0, T] \times \mathbb{R}^{3} \backslash \mathcal{K}\right)} \\
+\sum_{\substack{|\alpha|+m \leq 11 \\
m \leq 1}}\left\|\langle x\rangle^{-1} L^{m} \partial_{s, x}^{\alpha} w_{k}\right\|_{L_{s}^{2} L_{x}^{6}\left([0, T] \times \mathbb{R}^{3} \backslash \mathcal{K}\right)} \\
+\sum_{\substack{|\alpha|+m \leq 9 \\
m \leq 1}}\left\|\langle x\rangle^{-1} L^{m} Z^{\alpha} w_{k}\right\|_{L_{s}^{2} L_{x}^{6}\left([0, T] \times \mathbb{R}^{3} \backslash \mathcal{K}\right)} .
\end{gathered}
$$

If we let $M_{0}(T)$ denote the above quantity with $w_{k}$ replaced by $u_{0}$, then we note that (10.3) together with Lemma 3.4 implies

$$
\sup _{0<T<\infty} M_{0}(T) \leq C \varepsilon .
$$


We seek to find a constant $C_{1}$ so that for all $k$,

$$
M_{k}\left(T_{\varepsilon}\right) \leq C_{1} \varepsilon
$$

provided that $\varepsilon<\varepsilon_{0}$ and provided that $\varepsilon_{0}$ and the constant $\kappa$ occurring in the definition (1.8) are sufficiently small. To do this, we proceed inductively as in $\S 3$, and show that, provided $M_{k-1}\left(T_{\varepsilon}\right) \leq C_{1} \varepsilon$ and $\varepsilon \leq \kappa$, then

$$
M_{k}\left(T_{\varepsilon}\right) \leq C \cdot \varepsilon+C \cdot C_{1} \cdot \kappa \cdot\left(M_{k-1}\left(T_{\varepsilon}\right)+M_{k}\left(T_{\varepsilon}\right)\right),
$$

where $C$ is a universal constant. The bound (10.6) with $C_{1}=2 C$ follows from (10.7), provided $\kappa$ is sufficiently small.

We begin by estimating the fourth term in the formula for $M_{k}\left(T_{\varepsilon}\right)$, that is, the pointwise bounds for $Z^{\alpha} w_{k}$. By Theorem 4.1 and the support properties of $w_{k}$, this is bounded by

$$
\begin{aligned}
C \int_{0}^{T_{\varepsilon}} \int_{\mathbb{R}^{3} \backslash \mathcal{K}} \sum_{\substack{|\beta|+j \leq 8 \\
j \leq 1}}\left|L^{j} Z^{\beta} \square_{c} w_{k}(s, y)\right| \frac{d y d s}{|y|} \\
+C \int_{0}^{T_{\varepsilon}} \sum_{\substack{|\beta|+j \leq 5 \\
j \leq 1}}\left\|L^{j} \partial_{s, y}^{\beta} \square_{c} w_{k}(s, \cdot)\right\|_{L^{2}\left(\mathbb{R}^{3} \backslash \mathcal{K}\right)} d s .
\end{aligned}
$$

The contribution to (10.8) where $\square_{c} w_{k}$ is replaced by $\left[\square_{c}, \eta\right] u$ is bounded by $C \varepsilon$. Indeed, since this term is supported in the region $\frac{|y|}{2 c}<s<\frac{|y|}{c}$,

$$
\begin{gathered}
\int_{0}^{T_{\varepsilon}} \int_{\mathbb{R}^{3} \backslash \mathcal{K}} \sum_{\substack{|\beta|+j \leq 8 \\
j \leq 1}}\left|L^{j} Z^{\beta}\left[\square_{c}, \eta\right] u\right| \frac{d y d s}{|y|} \\
\leq C \int_{0}^{T_{\varepsilon}}\langle s\rangle^{-\frac{3}{4}} \int_{\mathbb{R}^{3} \backslash \mathcal{K}}\langle y\rangle^{-\frac{1}{4}-\frac{3}{2}} \sum_{\substack{|\beta|+j \leq 8 \\
j \leq 1}}\langle y\rangle^{\frac{3}{2}}\left|L^{j} Z^{\beta}\left[\square_{c}, \eta\right] u\right| d y d s \\
\leq C \sup _{0<t<\infty} \sum_{\substack{|\beta|+j \leq 8 \\
j \leq 1}}\left\|\langle y\rangle^{2} L^{j} Z^{\beta}\left[\square_{c}, \eta\right] u\right\|_{L^{2}\left(\mathbb{R}^{3} \backslash \mathcal{K}\right)},
\end{gathered}
$$

which by (10.3), and the homogeneity of $\eta$, is bounded by $C \varepsilon$. The contribution of $\left[\square_{c}, \eta\right] u$ to the second term in (10.8) is bounded using a similar argument,

$$
\begin{aligned}
& \int_{0}^{T_{\varepsilon}} \sum_{\substack{|\beta|+j \leq 5 \\
j \leq 1}}\left\|L^{j} \partial_{s, y}^{\beta}\left[\square_{c}, \eta\right] u(s, \cdot)\right\|_{L^{2}\left(\mathbb{R}^{3} \backslash \mathcal{K}\right)} d s \\
& \quad \leq C \int_{0}^{T_{\varepsilon}}\langle s\rangle^{-\frac{3}{2}} \sum_{\substack{|\beta|+j \leq 5 \\
j \leq 1}}\left\|\langle y\rangle^{\frac{3}{2}} L^{j} \partial_{s, y}^{\beta}\left[\square_{c}, \eta\right] u(s, \cdot)\right\|_{L^{2}\left(\mathbb{R}^{3} \backslash \mathcal{K}\right)} d s
\end{aligned}
$$

which is bounded by $C \varepsilon$ as argued for (10.9). 
The contribution to the expression (10.8) in which we replace the term $\square_{c} w_{k}$ by the term $(1-\eta) Q\left(d\left(u_{0}+w_{k-1}\right), d^{2}\left(u_{0}+w_{k}\right)\right)$ can be bounded by

$$
\begin{aligned}
& C \cdot\left(\varepsilon+\sum_{\substack{|\alpha|+m \leq 9 \\
m \leq 1}}\left\|\langle x\rangle^{-\frac{1}{2}} L^{m} Z^{\alpha} w_{k-1}^{\prime}\right\|_{L^{2}\left(\left[0, T_{\varepsilon}\right] \times \mathbb{R}^{3} \backslash \mathcal{K}\right)}\right) \\
& \times\left(\varepsilon+\sum_{\substack{|\alpha|+m \leq 9 \\
m \leq 1}}\left\|\langle x\rangle^{-\frac{1}{2}} L^{m} Z^{\alpha} w_{k-1}^{\prime}\right\|_{L^{2}\left(\left[0, T_{\varepsilon}\right] \times \mathbb{R}^{3} \backslash \mathcal{K}\right)}\right. \\
& \left.+\sum_{\substack{|\alpha|+m \leq 9 \\
m \leq 1}}\left\|\langle x\rangle^{-\frac{1}{2}} L^{m} Z^{\alpha} w_{k}^{\prime}\right\|_{L^{2}\left(\left[0, T_{\varepsilon}\right] \times \mathbb{R}^{3} \backslash \mathcal{K}\right)}\right) .
\end{aligned}
$$

For example, a typical term involving $u_{0}$ coming from the first term in 10.8 is handled as follows:

$$
\begin{aligned}
\int_{0}^{T_{\varepsilon}} \int_{\mathbb{R}^{3} \backslash \mathcal{K}} & \sum_{\substack{|\beta|+j \leq 8 \\
j \leq 1}}(1-\eta)\left|L^{j} Z^{\beta}\left(u_{0}^{\prime} w_{k}^{\prime \prime}\right)\right| \frac{d y d s}{|y|} \\
\leq & C \int_{0}^{T_{\varepsilon}}\langle s\rangle^{-\frac{3}{2}} \sum_{\substack{|\beta|+j \leq 9 \\
j \leq 1}}\left\|\langle y\rangle L^{j} Z^{\beta} u_{0}^{\prime}(s, \cdot)\right\|_{L^{2}\left(\mathbb{R}^{3} \backslash \mathcal{K}\right)} \\
& \times \sum_{\substack{|\beta|+j \leq 9 \\
j \leq 1}}\left\|\langle y\rangle^{-\frac{1}{2}} L^{j} Z^{\beta} w_{k}^{\prime}(s, \cdot)\right\|_{L^{2}\left(\mathbb{R}^{3} \backslash \mathcal{K}\right)} d s \\
\leq & C \sum_{\substack{|\beta|+j \leq 9 \\
j \leq 1}}\left\|\langle s\rangle^{-\frac{3}{2}}\langle y\rangle L^{j} Z^{\beta} u_{0}^{\prime}\right\|_{L^{2}\left(\left[0, T_{\varepsilon}\right) \times \mathbb{R}^{3} \backslash \mathcal{K}\right)}^{2} \\
& \times \sum_{\substack{|\beta|+j \leq 9 \\
j \leq 1}}\left\|\langle y\rangle^{-\frac{1}{2}} L^{j} Z^{\beta} w_{k}^{\prime}\right\|_{L^{2}\left(\left[0, T_{\varepsilon}\right) \times \mathbb{R}^{3} \backslash \mathcal{K}\right)} \\
\leq & C \sum_{\substack{|\beta|+j \leq 9 \\
j \leq 1}}\left\|\langle y\rangle^{-\frac{1}{2}} L^{j} Z^{\beta} w_{k}^{\prime}\right\|_{L^{2}\left(\left[0, T_{\varepsilon}\right) \times \mathbb{R}^{3} \backslash \mathcal{K}\right)}
\end{aligned}
$$

again using (10.3). Arguing as in (10.12), (10.9) and (10.10), one easily checks the bound (10.11) for the other terms in (10.8) involving $u_{0}$. To bound the contributions to (10.8) involving only $w_{k-1}, w_{k}$, we apply the Schwarz inequality to handle the first term in (10.8), and for the second term in (10.8), the bound (10.11) follows by applying Lemma 3.4 in the manner used to bound the first term on the right side of (3.18) (see (3.20)-(3.22)). We thus have the bound

$$
\begin{aligned}
& C\left(\varepsilon+\ln \left(T_{\varepsilon}\right)^{\frac{1}{2}} M_{k-1}\left(T_{\varepsilon}\right)\right) \times\left(\varepsilon+\ln \left(T_{\varepsilon}\right)^{\frac{1}{2}} M_{k-1}\left(T_{\varepsilon}\right)+\ln \left(T_{\varepsilon}\right)^{\frac{1}{2}} M_{k}\left(T_{\varepsilon}\right)\right) \\
& \leq C \cdot \varepsilon+C \cdot C_{1} \cdot \kappa \cdot\left(M_{k-1}\left(T_{\varepsilon}\right)+M_{k}\left(T_{\varepsilon}\right)\right) .
\end{aligned}
$$

Thus the fourth term in the definition of $M_{k}\left(T_{\varepsilon}\right)$ satisfies the bounds (10.7). 
All other terms in $M_{k}(t)$ occur in the left-hand side of (9.5), taking $N=10$, $w=w_{k}, t=T_{\varepsilon}$, and letting $\gamma$ be defined by

$$
\begin{aligned}
\left(\square_{\gamma} w_{k}\right)^{I} \equiv \square_{c_{I}} w_{k}^{I}-(1-\eta) \sum_{\substack{0 \leq i, j, l \leq 3 \\
1 \leq J, K \leq N}} B_{K, l}^{I J, i j}\left(\partial_{l} u_{0}^{K}+\partial_{l} w_{k-1}^{K}\right) \partial_{i} \partial_{j} w_{k}^{J} \\
=(1-\eta) B^{I}\left(d\left(u_{0}+w_{k-1}\right)\right)-\left[\square_{c_{I}}, \eta\right] u^{I} \\
\quad+(1-\eta) \sum_{\substack{0 \leq i, j, l \leq 3 \\
1 \leq J, K \leq N}} B_{K, l}^{I J, i j}\left(\partial_{l} u_{0}^{K}+\partial_{l} w_{k-1}^{K}\right) \partial_{i} \partial_{j} u_{0}^{J} .
\end{aligned}
$$

Hence, we need to show that each term on the right of $(9.5)$, with these values for $w_{k}, \gamma, t$ and $N$, can be dominated by the right-hand side of (10.7).

We first estimate

$$
\begin{aligned}
\int_{0}^{t}\left(\sum_{|\alpha| \leq 14}\left\|\square_{\gamma} \partial_{s, x}^{\alpha} w_{k}(s, \cdot)\right\|_{L^{2}\left(\mathbb{R}^{3} \backslash \mathcal{K}\right)}\right. & +\sum_{\substack{|\alpha|+m \leq 12 \\
m \leq 1}}\left\|\square_{\gamma} L^{m} \partial_{s, x}^{\alpha} w_{k}(s, \cdot)\right\|_{L^{2}\left(\mathbb{R}^{3} \backslash \mathcal{K}\right)} \\
& \left.+\sum_{\substack{|\alpha|+m \leq 10 \\
m \leq 1}}\left\|\square_{\gamma} L^{m} Z^{\alpha} w_{k}(s, \cdot)\right\|_{L^{2}\left(\mathbb{R}^{3} \backslash \mathcal{K}\right)}\right) d s .
\end{aligned}
$$

Consider the third term in (10.14), which is clearly bounded by

$$
\sum_{\substack{|\alpha|+m \leq 10 \\ m \leq 1}} \int_{0}^{t}\left(\left\|L^{m} Z^{\alpha} \square_{\gamma} w_{k}(s, \cdot)\right\|_{L^{2}\left(\mathbb{R}^{3} \backslash \mathcal{K}\right)}+\left\|\left[\square_{\gamma}, L^{m} Z^{\alpha}\right] w_{k}(s, \cdot)\right\|_{L^{2}\left(\mathbb{R}^{3} \backslash \mathcal{K}\right)}\right) d s .
$$

The contribution to $\square_{\gamma} w_{k}$ in the first term of [10.15) coming from $\left[\square_{c}, \eta\right] u$ is handled as in (10.10) above. To handle the contribution here from $(1-\eta) B^{I}\left(d\left(u_{0}+\right.\right.$ $\left.w_{k-1}\right)$ ), we bound those terms involving $u_{0}$ using (10.3) and arguing similar to (10.9), (10.10), and (10.12) above. In the same way, one bounds the contribution of the last term on the right side of (10.13) to the first term in (10.15). The contributions that remain to be bounded from both terms of (10.15) are identical to the first and third integrals on the right-hand side of (3.17), with $u_{k-1}^{\prime}, u_{k}^{\prime}$ there replaced by $w_{k-1}^{\prime}, w_{k}^{\prime}$, respectively. These terms can be estimated in an identical manner to that section, with the following remark in mind. Lemma 3.4 holds on our exterior domain for a general function $h$ (and the same proof applies without modification), but the analogue of estimate (3.11) of Lemma 3.5 on $\mathbb{R}^{3} \backslash \mathcal{K}$ requires Dirichlet boundary conditions to work. To get around this, we note that (3.11) holds on $\mathbb{R}^{3} \backslash \mathcal{K}$ provided either $\partial_{j}$ or the prime is a time derivative, by using the same argument as in the proof of Lemma 3.5. This similarly handles the case in which any factor of $Z^{\alpha}$ involves a time derivative. For the remaining cases, involving purely spatial derivatives, we can use the following elliptic estimate, which uses the 
fact that $w_{k}$ vanishes on the boundary,

$$
\begin{aligned}
& \sum_{\substack{|\alpha| \leq 9 \\
1 \leq i, j \leq 3}}\left\|\partial_{x}^{\alpha} \partial_{i} \partial_{j} w_{k}(t, \cdot)\right\|_{L^{2}\left(\mathbb{R}^{3} \backslash \mathcal{K}:|x|<1\right)} \\
& \quad \leq C \sum_{|\alpha| \leq 9}\left\|\partial_{x}^{\alpha} \Delta w_{k}(t, \cdot)\right\|_{L^{2}\left(\mathbb{R}^{3} \backslash \mathcal{K}:|x|<2\right)}+C\left\|w_{k}(t, \cdot)\right\|_{L^{6}\left(\mathbb{R}^{3} \backslash \mathcal{K}:|x|<2\right)} .
\end{aligned}
$$

The other two terms in (10.14) are estimated similarly, and thus these terms satisfy the bound (10.7).

Next consider the following terms from the right side of (9.5),

$$
\sum_{|\alpha| \leq 13}\left\|\square_{\gamma} \partial_{t, x}^{\alpha} w_{k}(t, \cdot)\right\|_{L^{2}\left(\mathbb{R}^{3} \backslash \mathcal{K}\right)}+\sum_{\substack{|\alpha|+m \leq 11 \\ m \leq 1}}\left\|\square_{\gamma} L^{m} \partial_{t, x}^{\alpha} w_{k}(t, \cdot)\right\|_{L^{2}\left(\mathbb{R}^{3} \backslash \mathcal{K}\right)} .
$$

We write

$$
\begin{aligned}
\left(\square_{\gamma} \partial_{t, x}^{\alpha} w_{k}\right)^{I}= & \partial_{t, x}^{\alpha} \square_{\gamma} w_{k}^{I}-\left[\partial_{t, x}^{\alpha}, \square_{\gamma}\right] w_{k}^{I} \\
= & -(1-\eta) \sum_{\substack{0 \leq i, j, l \leq 3 \\
1 \leq J, K \leq N}}^{\substack{I J, i j \\
K, l}}\left[\partial_{t, x}^{\alpha}, \partial_{l} u_{0}^{K}+\partial_{l} w_{k-1}^{K}\right] \partial_{i} \partial_{j} w_{k}^{J} \\
& +\partial_{t, x}^{\alpha}(1-\eta) \sum_{\substack{0 \leq i, j, l \leq 3 \\
1 \leq J, K \leq N}} B_{K, l}^{I J, i j}\left(\partial_{l} u_{0}^{K}+\partial_{l} w_{k-1}^{K}\right) \partial_{i} \partial_{j} u_{0} \\
& \quad+\partial_{t, x}^{\alpha}(1-\eta) B^{I}\left(d\left(u_{0}+w_{k-1}\right)\right)-\partial_{t, x}^{\alpha}\left[\square_{c_{I}}, \eta\right] u^{I} .
\end{aligned}
$$

Upon expansion, each term on the right-hand side, with the exception of the last, which has $L^{2}$ norm bounded by $C \varepsilon$ by (10.3), is the product of two terms, at least one of which involves at most 8 derivatives. We can obtain $L^{\infty}$ bounds on such a term by Sobolev embedding, and hence estimate the $L^{2}$ norm of the product by the right-hand side of (10.7). The same argument applies to the second term in (10.16).

Finally, consider

$$
\begin{aligned}
\sum_{|\alpha| \leq 12}\left\|\square_{c} \partial_{s, x}^{\alpha} w_{k}\right\|_{L^{2}\left(\left[0, T_{\varepsilon}\right] \times \mathbb{R}^{3} \backslash \mathcal{K}\right)}+\sum_{\substack{|\alpha|+m \leq 10 \\
m \leq 1}}\left\|\square_{c} L^{m} \partial_{s, x}^{\alpha} w_{k}\right\|_{L^{2}\left(\left[0, T_{\varepsilon}\right] \times \mathbb{R}^{3} \backslash \mathcal{K}\right)} & +\sum_{\substack{|\alpha|+m \leq 8 \\
m \leq 1}}\left\|\square_{c} L^{m} Z^{\alpha} w_{k}\right\|_{L^{2}\left(\left[0, T_{\varepsilon}\right] \times \mathbb{R}^{3} \backslash \mathcal{K}\right)} .
\end{aligned}
$$

We write

$$
\begin{aligned}
\square_{c} \partial_{t, x}^{\alpha} w_{k}^{I}=\partial_{t, x}^{\alpha} & \sum_{\substack{0 \leq i, j, l \leq 3 \\
1 \leq J, K \leq N}} B_{K, l}^{I J, i j}\left(\partial_{l} u_{0}^{K}+\partial_{l} w_{k-1}^{K}\right) \partial_{i} \partial_{j} w_{k}^{J} \\
& +\partial_{t, x}^{\alpha} \sum_{\substack{0 \leq i, j, l \leq 3 \\
1 \leq J, K \leq N}} B_{K, l}^{I J, i j}\left(\partial_{l} u_{0}^{K}+\partial_{l} w_{k-1}^{K}\right) \partial_{i} \partial_{j} u_{0} \\
& +\partial_{t, x}^{\alpha} B^{I}\left(d\left(u_{0}+w_{k-1}\right)\right)-\partial_{t, x}^{\alpha}\left[\square_{c_{I}}, \eta\right] u^{I} .
\end{aligned}
$$

The last term involving $u$ is easily handled as in (10.9) above. As a representative of the other terms that arise upon expanding derivatives, noting that $|\alpha| \leq 12$, 
consider

$$
\sum_{|\beta| \leq 6,|\mu| \leq 13}\left\|\partial_{t, x}^{\beta} w_{k-1}^{\prime} \partial_{t, x}^{\mu} w_{k}^{\prime}\right\|_{L^{2}\left(\left[0, T_{\varepsilon}\right] \times \mathbb{R}^{3} \backslash \mathcal{K}\right)} .
$$

We now apply Lemma 3.4 to the $\beta$ terms and sum over $R$ to conclude that

$$
\begin{aligned}
& \quad \sum_{|\beta| \leq 6,|\mu| \leq 13}\left\|\partial_{t, x}^{\beta} w_{k-1}^{\prime} \partial_{t, x}^{\mu} w_{k}^{\prime}\right\|_{L^{2}\left(\left[0, T_{\varepsilon}\right] \times \mathbb{R}^{3} \backslash \mathcal{K}\right)} \\
& \leq C \sum_{|\beta| \leq 8}\left\|\langle x\rangle^{-1} Z^{\beta} w_{k-1}^{\prime}\right\|_{L^{2}\left(\left[0, T_{\varepsilon}\right] \times \mathbb{R}^{3} \backslash \mathcal{K}\right)} \sup _{0<t<T_{\varepsilon}} \sum_{|\mu| \leq 13}\left\|\partial_{t, x}^{\mu} w_{k}^{\prime}(t, \cdot)\right\|_{L^{2}\left(\mathbb{R}^{3} \backslash \mathcal{K}\right)} \\
& \leq C \cdot C_{1} \cdot \varepsilon \cdot \ln \left(T_{\varepsilon}\right)^{\frac{1}{2}} M_{k}\left(T_{\varepsilon}\right) \leq C \cdot C_{1} \cdot \kappa \cdot M_{k}\left(T_{\varepsilon}\right) .
\end{aligned}
$$

The other terms are similarly seen to be bounded by the right-hand side of (10.7), which completes the proof of (10.7).

Next, using the energy inequality, one observes that $\left\{w_{k}\right\}$ is a Cauchy sequence in the energy norm. Because of this and (10.6) we conclude that $w_{k}$ must converge to a solution of (10.5) that satisfies the bounds in (10.6). Consequently, $u=u_{0}+w$ will be a solution of the original equation (1.2), which verifies the analogue of (10.6). If the data is $C^{\infty}$ and satisfies the compatibility conditions to infinite order, the solution will be $C^{\infty}$ on $\left[0, T_{\varepsilon}\right] \times \mathbb{R}^{3} \backslash \mathcal{K}$ by standard local existence theory (see, e.g., [10]).

This completes the proof of Theorem 1.1 .

\section{ACKNOWLEDGMENTS}

The authors were supported in part by the NSF. The authors would like to thank the referee for a number of helpful suggestions and comments. In particular, we are grateful for the suggestion that non-diagonal systems should be handled. The third author would like to thank J.-M. Delort for helpful conversations regarding this paper. He would also like to thank Paris Nord for their hospitality during a recent visit where part of this research was carried out.

\section{REFERENCES}

[1] D. Christodoulou: Global solutions of nonlinear hyperbolic equations for small initial data, Comm. Pure Appl. Math. 39, (1986), 267-282. MR 87c:35111

[2] P. S. Datti: Nonlinear wave equations in exterior domains, Nonlinear Anal. 15 (1990), 321-331. MR 91k:35163

[3] D. Gilbarg and N. Trudinger: Elliptic partial differential equations of second order, Springer-Verlag, Second edition, Third Printing, 1998. MR 86c:35035

[4] P. Godin: Global existence of solutions to some exterior radial quasilinear Cauchy-Dirichlet problems, Amer. J. Math. 117 (1995), 1475-1505. MR 97j:35096

[5] N. Hayashi: Global existence of small solutions to quadratic nonlinear wave equatinos in an exterior domain, J. Funct. Anal. 131 (1995), 302-344. MR 96f:35113

[6] L. Hörmander: Lectures on nonlinear hyperbolic equations, Springer-Verlag, Berlin, 1997. MR 98e:35103

[7] L. Hörmander: $L^{1}, L^{\infty}$ estimates for the wave operator, Analyse Mathématique et Applications, Gauthier-Villars, Montrouge, 1988, pp. 211-234. MR 90e:35113

[8] F. John: Nonlinear wave equations, formation of singularities, American Mathematical Society, Providence, RI, 1989. MR 91g:35001

[9] F. John and S. Klainerman: Almost global existence to nonlinear wave equations in three dimensions Comm. Pure Appl. Math. 37 (1984), 443-455. MR 85k:35147

[10] M. Keel, H. Smith, and C. D. Sogge: Global existence for a quasilinear wave equation outside of star-shaped domains, J. Funct. Anal. 189 (2002), 155-226. MR 2003f:35209 
[11] M. Keel, H. Smith, and C. D. Sogge: Almost global existence for some semilinear wave equations, J. d'Anal. Math. 87 (2002), 265-279.

[12] S. Klainerman: Uniform decay estimates and the Lorentz invariance of the classical wave equation, Comm. Pure Appl. Math. 38 (1985), 321-332. MR 86i:35091

[13] S. Klainerman: The null condition and global existence to nonlinear wave equations, Lectures in Applied Math. 23 (1986), 293-326. MR 87h:35217

[14] S. Klainerman and T. Sideris: On almost global existence for nonrelativistic wave equations in 3D, Comm. Pure Appl. Math. 49 (1996), 307-321. MR 96m:35231

[15] M. Kovalyov: Long-time behavior of solutions of a system of nonlinear wave equations, Comm. Partial Differential Equations 12 (1987), 471-501. MR 88c:35100

[16] P. D. Lax, C. S. Morawetz, and R. S. Phillips: Exponential decay of solutions of the wave equation in the exterior of a star-shaped obstacle, Comm. Pure Appl. Math. 16 (1963), 477-486. MR 27:5033

[17] P. D. Lax and R. S. Phillips: Scattering theory, revised edition, Academic Press, San Diego, 1989. MR 90k:35005

[18] C. S. Morawetz: The decay of solutions of the exterior initial-boundary problem for the wave equation, Comm. Pure Appl. Math. 14 (1961), 561-568. MR 24:A2744

[19] C. S. Morawetz: Time decay for the nonlinear Klein-Gordon equation, Proc. Roy. Soc. A 306 (1968), 291-296. MR 38:2455

[20] C. S. Morawetz and W. Strauss: Decay and scattering of solutions of a nonlinear relativistic wave equation, Comm. Pure Appl. Math. 25 (1972), 1-31. MR 46:2239

[21] C. S. Morawetz, J. Ralston, and W. Strauss: Decay of solutions of the wave equation outside nontrapping obstacles, Comm. Pure Appl. Math. 30 (1977), 447-508. MR 58:23091a

[22] T. Sideris: Nonresonance and global existence of prestressed nonlinear elastic waves, Ann. of Math. 151 (2000), 849-874. MR 2001g:35180

[23] T. Sideris: The null condition and global existence of nonlinear elastic waves, Invent. Math. 123 (1996), 323-342. MR 97a:35158

[24] T. Sideris and S. Y. Tu: Global existence for systems of nonlinear wave equations in $3 D$ with multiple speeds, SIAM J. Math. Anal. 33 (2001), 477-488. MR 2002j:35220

[25] H. Smith and C. D. Sogge: Global Strichartz estimates for nontrapping perturbations of the Laplacian, Comm. Partial Differential Equations 25 (2000), 2171-2183. MR 2001j:35180

[26] C. D. Sogge: Lectures on nonlinear wave equations, International Press, Boston, MA, 1995. MR 2000g:35153

[27] C. D. Sogge: Global existence for nonlinear wave equations with multiple speeds, Proceedings of the 2001 Mount Holyoke Conference on Harmonic Analysis, pp. 353-366, Contemp. Math. 320, Amer. Math. Soc., Providence, RI, 2003.

[28] Y. Shibata and Y. Tsutsumi: On a global existence theorem of small amplitude solutions for nonlinear wave equations in an exterior domain, Math. Z. 191 (1986), 165-199. MR 87i:35122

[29] K. Yokoyama: Global existence of classical solutions to systems of wave equations with critical nonlinearity in three space dimensions, J. Math. Soc. 52 (2000), 609-632. MR 2002a:35156

Department of Mathematics, University of Minnesota, Minneapolis, Minnesota 55455

Department of Mathematics, University of Washington, Seattle, Washington 98195

Department of Mathematics, The Johns Hopkins University, Baltimore, Maryland 21218 